\title{
EV-associated miRNAs from peritoneal lavage as potential diagnostic biomarkers in colorectal cancer
}

\author{
Berta Roman-Canal 1,2,3, Jordi Tarragona ${ }^{1}$, Cristian Pablo Moiola ${ }^{1,4}$, Sònia Gatius ${ }^{1}$, Sarah Bonnin ${ }^{5}$, \\ Maria Ruiz-Miró ${ }^{1}$, José Enrique Sierra ${ }^{6}$, Maria Rufas $^{6}$, Esperanza González ${ }^{7}$, José M. Porcel ${ }^{8}$, \\ Antonio Gil-Moreno ${ }^{4,9}$, Juan M. Falcón-Pérez ${ }^{7,10}$, Julia Ponomarenko ${ }^{5,11}$, Xavier Matias-Guiu 1,2,12* \\ and Eva Colas ${ }^{4 *}$ (1)
}

\begin{abstract}
Background: Colorectal cancer (CRC) is the third leading cause of cancer-related mortality worldwide. Current systematic methods for diagnosing have inherent limitations so development of a minimally-invasive diagnosis, based on the identification of sensitive biomarkers in liquid biopsies could therefore facilitate screening among population at risk.

Methods: In this study, we aim to develop a novel approach to identify highly sensitive and specific biomarkers by investigating the use of extracellular vesicles (EVs) isolated from the peritoneal lavage as a source of potential miRNA diagnostic biomarkers. We isolated EVs by ultracentrifugation from 25 ascitic fluids and 25 peritoneal lavages from non-cancer and CRC patients, respectively. Analysis of the expression of EV-associated miRNAs was performed using Taqman OpenArray technology through which we could detect 371 miRNAs.

Results: 210 miRNAs were significantly dysregulated (adjusted $p$ value $<0.05$ and abs(logFC) $\geq 1$ ). The top-10 miRNAs, which had the AUC value higher than 0.95, were miRNA-199b-5p, miRNA-150-5p, miRNA-29c-5p, miRNA-218-5p, miRNA-99a-3p, miRNA-383-5p, miRNA-199a-3p, miRNA-193a-5p, miRNA-10b-5p and miRNA-181c-5p.
\end{abstract}

Conclusions: This finding opens the avenue to the use of EV-associated miRNA of peritoneal lavages as an untapped source of biomarkers for CRC.

Keywords: Colorectal cancer, Biomarkers, Diagnostic, miRNAs, Ascitic fluid, Peritoneal lavage, Liquid biopsy, Extracellular vesicles, Colon cancer

\section{Background}

Colorectal cancer (CRC) is the third most common type of malignant tumor and the third leading cause of cancer-related mortality worldwide among men and

\footnotetext{
*Correspondence: fjmatiasguiu.lleida.ics@gencat.cat; eva.colas@vhir.org

${ }^{+}$Xavier Matias-Guiu and Eva Colas are senior co-authors

${ }^{4}$ Biomedical Research Group in Gynecology, Vall Hebron Research

Institute (VHIR), Universitat Autònoma de Barcelona, CIBERONC, Pg. Vall Hebron 119-129, 08035 Barcelona, Spain ${ }^{12}$ Oncologic Pathology Group, Department of Medicine UdL, Biomedical Research Institute of Lleida (IrbLleida), Av. Rovira Roure 80, 25198 Lleida, Spain

Full list of author information is available at the end of the article
}

women [1]. The overall survival of colorectal cancer is $65 \%$, but this is highly dependent upon the disease stage at diagnosis, and ranges from a $90 \%$ of 5 -year survival rate for cancers detected at the localized stage $(40 \%$ of the cases) and $70 \%$ for regional (35\% of the cases) to $15 \%$ for distant metastatic cancer (20\% of the cases) [2]. Current systematic methods for diagnosis, such as fecal occult blood test and flexible sigmoidoscopy, help to reduce mortality by removing precursor lesions and making diagnosis at an earlier stage. However, these techniques have inherent limitations, such as low sensitivity and invasiveness for patients, respectively; and 
the burden of disease and mortality is still high [3]. Serum tumor markers CA19-9 and CEA have been used for detection of many types of cancer, but their sensitivity for the detection of CRC is low [4]. Therefore, development of a minimally-invasive diagnosis, based on the identification of sensitive biomarkers in liquid biopsies could therefore facilitate screening among population at risk of CRC, impact on early detection, and thus, decrease CRC-related mortality.

MiRNAs are a highly conserved family of endogenous non-coding and single-stranded RNAs that are 19-24 nucleotides in length [5]. Generally, miRNAs negatively regulate gene expression via binding to the $3^{\prime}$-untranslated region ( $3^{\prime}$-UTR) of their target double-stranded mRNA that results in transcriptional repression or mRNA degradation upon dicer complex [6]. miRNAs have been implicated in development and progression of CRC by functioning as oncogenes and tumor suppressors [7]. Recent studies demonstrated that miRNAs are secreted from various cells, including cancer cells, into bodily fluids such as blood, urine, breast milk, and saliva, either as free miRNAs or via extracellular vesicles (EVs) [4].

EVs are $20-200 \mathrm{~nm}$ membrane vesicles released by either directly from plasma membranes, or from intracellular multivesicular bodies by their fusion with the cell membrane. Their function is to mediate intercellular communication, influencing the recipient cell function. Importantly, EVs have awakened the interest of the scientific community as a source of biomarkers, mainly because they carry a broad range of bioactive material (proteins, metabolites, RNA, miRNA, etc.) and this material is well-protected owing to the EVs lipid bilayer membrane, even if EVs are extracted from circulating or proximal body fluids [8].

Herein, we investigated the use of EVs isolated from the peritoneal lavage, a proximal fluid in CRC patients, as a source of potential diagnostic biomarkers. To do so, we conducted miRNA-profiling of EVs isolated from peritoneal lavages of surgical CRC patients and ascitic fluids of non-cancer patients by using the TaqMan OpenArray Human MicroRNA Panel. We unveiled the most relevant individual miRNAs for diagnosing CRC and characterized the biological and molecular landscape of the CRC milieu. The study was conceived as a proof of concept investigation to demonstrate the feasibility of peritoneal lavage as a source of EV-associated miRNAs in patients with CRC.

\section{Methods}

Patients and ascitic fluid and peritoneal lavages collection Participants in the study attended to the Hospital Arnau de Vilanova in Lleida, Spain. The Clinical Research
Ethics Committee of the hospital approved the study and all the participating patients provided a signed informed consent. Ascitic fluids and peritoneal lavages were extracted from a cohort of 50 patients, corresponding to 25 control patients with decompensated cirrhosis, and 25 patients with CRC who underwent curative surgery. In control patients, the collection of ascitic fluid was aspirated using 18 or $21 \mathrm{G}$ needles (for diagnostic paracentesis) or an over-the-needle catheter device (for therapeutic paracentesis). The procedure was performed under sterile conditions, the site of needle insertion was selected by ultrasound guidance, and skin and parietal peritoneum were previously anesthetized with $2 \%$ mepivacine. A total of $100 \mathrm{~mL}$ of ascitic fluid was gently aspirated, collected into a $50 \mathrm{~mL}$ tube and stored at $-80{ }^{\circ} \mathrm{C}$. In CRC patients, the collection of peritoneal lavage was performed before the surgery, once the abdominal cavity has been opened and prior to any manipulation of the colon. A total of $100 \mathrm{~mL}$ of physiological saline were instilled into the abdominal cavity with a $50 \mathrm{~mL}$ syringe, mobilizing patients for the correct distribution of saline, which was then extracted with a $50 \mathrm{~mL}$ syringe connected to a 14-gauge aspiration needle. The peritoneal lavage was gently aspirated. A volume ranging from 50 to $100 \mathrm{~mL}$ was collected and stored at $-80^{\circ} \mathrm{C}$. The clinical features of each patient are listed in Additional file 1: Table S1.

\section{EVs isolation}

EVs were isolated with a differential centrifugation method as previously described [9] with slight modifications. Briefly, ascitic fluids and peritoneal lavages were centrifuged at $300 \times g$ for $10 \mathrm{~min}$, followed by a centrifugation at $2500 \times g$ for $20 \mathrm{~min}$ and a centrifugation of $10,000 \mathrm{~g}$ for $30 \mathrm{~min}$ (Thermo Scientific Heraeus MultifugeX3R Centrifuge (FiberLite rotor F15-8x-50c)). The supernatant was then filtered through $0.22 \mu \mathrm{m}$ filters (Merck Millipore) and the sample obtained was transferred to ultracentrifuge tubes (Beckman Coulter) and filled with PBS to perform two consecutive ultracentrifugation steps at $100,000 \mathrm{~g}$ for $2 \mathrm{~h}$ each on a Thermo Scientific Sorvall WX UltraSeries Centrifuge with an AH-629 rotor. The pellet containing the EVs was resuspended in $50 \mu \mathrm{L}$ of PBS. From those, $5 \mu \mathrm{L}$ were isolated for nanoparticle tracking analysis (NTA) and quantification, and the rest was frozen at $-80{ }^{\circ} \mathrm{C}$ with $500 \mu \mathrm{L}$ of Qiazol for RNA extraction.

\section{Nanoparticle tracking analysis}

Size and number of EVs was determined using a Nanosight LM10 instrument equipped with a $405 \mathrm{~nm}$ laser and a Hamamatsu C11440 ORCA-Flash 2.8 camera 
(Hamamatsu) with Nanoparticle Tracking Analysis (NTA, Malvern Instruments, UK). Each sample was diluted appropriately with Milli-Q water (Milli-Q Synthesis, Merck Millipore, Massachusetts, USA) to give counts in the linear range of the instrument. The particles in the laser beam undergo Brownian motion, and a video was recorded for $60 \mathrm{~s}$ in triplicate. Analysis was performed following manufacturer's instructions and data were analyzed using the version 2.3 of the NTA-software.

\section{Total RNA extraction and OpenArray analysis}

Total RNA, including miRNAs and other RNAs, was isolated from the EVs samples using the miRNeasy MiniKit (Qiagen) according to manufacturer's protocol. RNA from EVs was eluted with $30 \mu \mathrm{L}$ of Nuclease-free water (Ambion). MiRNA expression was performed using TaqMan OpenArray Human MicroRNA Panel, QuantStudio 12 K Flex (Catalog number: 4470187, Thermo Fisher Scientific), a fixed-content panel containing 754 well-characterized human miRNA sequences from the Sanger miRBase v14 and according to the manufacturer's instructions. Reverse transcription (RT) was performed on $2 \mu \mathrm{L}$ RNA using Megaplex ${ }^{\mathrm{TM}}$ Primer Pools A and B and the supporting TaqMan ${ }^{\circledR}$ MicroRNA Reverse Transcription Kit as follows: $15 \mathrm{~min}$ at $42{ }^{\circ} \mathrm{C}$ and $5 \mathrm{~min}$ at $85^{\circ} \mathrm{C}$. Then, $5 \mu \mathrm{L}$ of the resulting cDNA was preamplified prior to real-time PCR analysis using Megaplex ${ }^{\mathrm{TM}}$ PreAmp Pools and the TaqMan ${ }^{\circledR}$ PreAmp Master Mix using the following conditions: one single step at $95{ }^{\circ} \mathrm{C}$ during $5 \mathrm{~min}, 20$ cycles of a two-steps program $\left(3 \mathrm{~s}, 95^{\circ} \mathrm{C}\right.$ and $30 \mathrm{~s}, 60^{\circ} \mathrm{C}$ ) followed by a single cycle of $10 \mathrm{~min}$ at $99^{\circ} \mathrm{C}$ to inactivate the enzyme. The preamplified products were diluted 1:20 in 0.1 $\times$ TE buffer pH8.0, and mixed in 1:1 with TaqMan ${ }^{\circledR}$ OpenArray ${ }^{\circledR}$ Real-Time PCR Master Mix in the 384-well OpenArray ${ }^{\circledR}$ Sample Loading Plate. TaqMan ${ }^{\circledR}$ OpenArray ${ }^{\circledR}$ MicroRNA Panels were automatically loaded using the AccuFill ${ }^{\mathrm{TM}}$ System.

\section{Preprocessing and differential expression analysis}

All bioinformatics analysis was performed with the BioConductor (version 3.7) [10] project in the $R$ statistical environment (version 3.5.0) [11]. For the data preprocessing, the HTqPCR (version 1.34) R package [12] was used. Probes that had a "Cycle threshold" (Ct) value of 40 in all samples were removed. Further samples in which more than $80 \%$ of the probes had a Ct value above 40 were retained. To assure comparability across samples, the $\mathrm{Ct}$ values were delta normalized. The average $\mathrm{Ct}$ values of the probes $h s a-m i R-150-5 p$, hsa-let $-7 g-5 p$, $h s a-m i R-598-3 p$, and $h s a-m i R-361-3 p$ were used for normalization. These probes had the $\mathrm{Ct}$ values of 40 in a maximum of three samples, and the lowest interquartile range across samples. Differential expression analysis was carried out with an empirical Bayes approach on linear models, using the limma (version 3.36) R Package [13]. Results were corrected for multiple testing using the False Discovery Rate (FDR) [14].

\section{Development of predictors}

For predictive analysis, the whole patient cohort was randomly divided into training and validation sets with the 3:2 ratio. Calculated (with the limma $\mathrm{R}$ Package) relative miRNA expression values were used as input variables to a logistic regression model between groups. Each miRNA (adjusted p-value $<0.05$ ) was fitted in the logistic regression model to differentiate the $\mathrm{CRC}$ and the control patients groups in the training set and its classification ability was evaluated using the AUC (area under the ROC curve), accuracy, sensitivity, and specificity values on the validation set. The procedure from division into training and validation sets and fitting the logistic model was repeated 500 times and statistics were collected.

miRNA target genes prediction and bioinformatics analysis miRNAs target genes were obtained using the Predictive Target Module of miRWalk2.0 online software [15] (https://goo.gl/ajG9ja), considering the following parameters: 3'UTR localization, miRNA seed start at position 1 and minimum 7 bp seed length. To improve the accuracy of target gene prediction, only those transcripts that were predicted in at least 8 out of the 12 databases were considered (miRWalk, miRanda, MicroT4, miRDB, miRMap, miRBridge, miRNAMap, PICTAR2, RNA22, PITA, TargetScan, and RNAhybrid). Gene Ontology (GO) functional analysis were used to analyze the potential functions of the predicted target genes, using the online Panther software [16] (http://www.pantherdb.org/). Biological process (BP) and molecular function (MF) GO terms were analyzed and plotted.

\section{Results}

We analyzed the miRNA profile of EVs isolated from the ascitic fluid of 25 control individuals and peritoneal lavage of 25 CRC patients. Additional file 2: Figure S1 illustrates the workflow that was followed in this study. The quality of EVs isolated from the ascitic fluids and peritoneal lavages was measured by size distribution and concentration by Nanoparticle Tracking Analysis, demonstrating that we analyzed a population mostly enriched in small EVs but also containing a low representation of larger vesicles (Additional file 3: Figure S2). MiRNAs were extracted from EVs for a systematic miRNA expression analysis using the Taqman OpenArray technology, through which we detected 371 out of the 754 miRNAs (49.2\%) present in the OpenArray. Probes that had the 
Ct value of 40 in all samples and samples in which more than $80 \%$ of the probes had the Ct value above 40 were removed, resulting in 355 miRNAs from 22 control and 19 CRC patients analyzed for the differential expression analysis (Table 1 ).

The differential expression analysis between cancer and control cases yielded a list of 210 miRNAs that were significantly dysregulated (adj. p-value $<0.05$ and $\operatorname{logFC}$ lower or higher than 1). Among those, 207 miRNA were found to be downregulated and 3 miRNA were upregulated in CRC patients. To evaluate whether these miRNAs can be used as diagnostics biomarkers, we performed a predictive analysis using the logistic modeling. Ten miRNAs demonstrated predictive performance at the AUC values higher than 0.95: miRNA-199b-5p, miRNA150-5p, miRNA-29c-5p, miRNA-218-5p, miRNA-99a-3p, miRNA-383-5p, miRNA-199a-3p, miRNA-193a-5p, miRNA-10b-5p and miRNA-181c-5p (Table 2; Fig. 1). All those miRNAs were downregulated from 3.52 to 12.82 in the $\log _{2}$ scale with adjusted p-value lower than $1.56 \mathrm{E}-05$, except miRNA-150-5p which was upregulated (adjusted p-value 3.41E-04). In Table 3, studies reporting an association between each of these top-10 miRNAs and CRC are described based on a search of Pubmed for each miRNA and the word "colorectal cancer". Although there are some controversies among the different studies, most of the miRNA dysregulations observed in our study are concordant with the observations reported by other authors, either in tissue, plasma or stool samples. MiRNA-199b-5p, miRNA-29c-5p, and miRNA-99a-3p have never been reported previously in association to CRC.

Table 1 Clinicopathological characteristics of patients

\begin{tabular}{lcc}
\hline & Colon Cancer & Control \\
\hline Age & 74 & 65 \\
Median & 50 & 52 \\
Minimum & 88 & 90 \\
Maximum & & \\
Gender & 12 & 4 \\
Female & 7 & 18 \\
Male & & \\
Pathology & 19 & - \\
Colon cancer & 15 & - \\
ADC low grade & 4 & - \\
ADC other types & - & 20 \\
Hepatic cirrhosis & - & 2 \\
Others &
\end{tabular}

Clinical characteristics of the final cohort of patients included in the study after data normalization

$A D C$ adenocarcinoma
To further understand the milieu generated by CRC EVs, we performed a bioinformatics study to first unveil the predicted transcripts that are regulated by all the differential miRNAs, and then assess the biological processes and molecular functions in which they participate. A total of 9358 transcripts were found to be regulated by the 210 miRNA differentially expressed. Figure 2 shows the number and most frequently regulated transcripts predicted for each dysregulated miRNA. To comprehensively integrate the properties of all target transcripts, they were studied using Gene Ontology (GO). The most enriched biological processes in CRC EVs were metabolic processes (24.3\%), mostly including biosynthetic process, organic substance metabolomic process and cellular metabolic process; biological regulation (22.5\%); cellular processes (10.7\%), signal transduction, cellular component organization and cellular metabolic process; and cellular component organization or biogenesis, including cellular component organization (Fig. 3a). In relation to the most altered molecular functions in CRC EVs, the Gene Ontology (GO) analysis revealed that many targeted transcripts were found to be involved in binding (37.8\%), including protein binding and organic cyclic compound binding; and in catalytic activity (31.2\%), including catalytic activity, and protein and hydrolase activity (Fig. 3b).

\section{Discussion}

In this study we investigated, for the first time, the miRNA content of EVs isolated from peritoneal lavages and ascitic liquid of CRC and control patients, respectively. Our study shows that EV-associated miRNAs can be consistently extracted from peritoneal lavages and ascitic liquids and that miRNA expression profiles can indicate and represent the status of CRC patients. The EV-associated miRNA were analyzed by Taqman OpenArray technology and the differential expression analysis yielded a list of 210 miRNAs that were significantly dysregulated in CRC patients, being downregulated the $98.57 \%$ of the altered miRNAs.

The finding that miRNAs are dysregulated in CRC patients is known, as many studies have reported this previously, mostly in tissue specimens [17] but also in different body fluids. In CRC, most of the studies use plasma or serum as it is the most common, easyto-handle, accessible liquid biopsy. The first report detected 69 miRNAs in serum of CRC patients but not in serum of normal controls [18]. Since then, several studies have identified miRNA upregulation or downregulation in plasma or serum samples [17] including studies that have focus on the search of biomarkers in miRNAs dysregulated in the vesicular fraction of the serum or plasma of CRC patients. Hiroko OgataKawata et al. [4] analyzed the EV-associated miRNA 
Table 2 miRNA transcripts displaying a significant differential expression in patients with CRC compared to control patients

\begin{tabular}{|c|c|c|c|c|c|c|c|c|c|}
\hline miRNA & LogFC & p-value & Adj. p-value & AUC & $\begin{array}{l}\text { AUC 95\% } \\
\text { Cl_lower }\end{array}$ & $\begin{array}{l}\text { AUC 95\% } \\
\text { Cl_upper }\end{array}$ & Accuracy & Sensitivity & Specificity \\
\hline hsa-miR-199b-5p_478486_mir & -12.82 & $2.59 \mathrm{E}-08$ & $9.85 \mathrm{E}-07$ & 1 & 1 & 1 & 0.967 & 0.968 & 0.964 \\
\hline hsa-miR-150-5p_477918_mir & 2.58 & $7.10 \mathrm{E}-05$ & $3.41 \mathrm{E}-04$ & 0.978 & 0.959 & 0.996 & 0.919 & 0.936 & 0.899 \\
\hline hsa-miR-29c-5p_478005_mir & -2.93 & $2.78 \mathrm{E}-08$ & $9.85 \mathrm{E}-07$ & 0.973 & 0.954 & 0.991 & 0.943 & 0.943 & 0.944 \\
\hline hsa-miR-218-5p_477977_mir & -8.16 & $6.51 \mathrm{E}-07$ & $8.25 E-06$ & 0.97 & 0.945 & 0.995 & 0.913 & 0.905 & 0.921 \\
\hline hsa-miR-99a-3p_479224_mir & -4.89 & $1.51 \mathrm{E}-08$ & $8.95 \mathrm{E}-07$ & 0.97 & 0.95 & 0.99 & 0.94 & 0.976 & 0.9 \\
\hline hsa-miR-383-5p_478079_mir & -8.33 & $3.55 \mathrm{E}-15$ & $1.26 \mathrm{E}-12$ & 0.968 & 0.952 & 0.985 & 0.939 & 0.94 & 0.938 \\
\hline hsa-miR-199a-3p_477961_mir & -6.16 & $2.84 \mathrm{E}-09$ & $2.65 \mathrm{E}-07$ & 0.968 & 0.942 & 0.994 & 0.905 & 0.92 & 0.887 \\
\hline hsa-miR-193a-5p_477954_mir & -3.62 & $1.32 \mathrm{E}-06$ & $1.56 \mathrm{E}-05$ & 0.962 & 0.932 & 0.991 & 0.873 & 0.852 & 0.897 \\
\hline hsa-miR-10b-5p_478494_mir & -2.79 & $2.58 \mathrm{E}-07$ & 4.17E-06 & 0.957 & 0.93 & 0.983 & 0.871 & 0.875 & 0.866 \\
\hline hsa-miR-181c-5p_477934_mir & -3.52 & $1.23 \mathrm{E}-05$ & $8.74 \mathrm{E}-05$ & 0.952 & 0.929 & 0.974 & 0.833 & 0.859 & 0.803 \\
\hline hsa-miR-708-5p_478197_mir & -6.45 & $9.72 \mathrm{E}-08$ & $2.29 \mathrm{E}-06$ & 0.946 & 0.917 & 0.975 & 0.877 & 0.834 & 0.926 \\
\hline hsa-miR-125b-5p_477885_mir & -2.35 & $9.27 \mathrm{E}-07$ & $1.13 \mathrm{E}-05$ & 0.946 & 0.918 & 0.974 & 0.885 & 0.884 & 0.885 \\
\hline hsa-miR-140-5p_477909_mir & -5.82 & $4.59 E-05$ & $2.39 E-04$ & 0.943 & 0.919 & 0.968 & 0.817 & 0.825 & 0.807 \\
\hline hsa-miR-451a_478107_mir & -8.35 & $1.34 \mathrm{E}-07$ & $2.64 \mathrm{E}-06$ & 0.942 & 0.913 & 0.972 & 0.843 & 0.881 & 0.8 \\
\hline hsa-miR-148b-3p_477824_mir & -3.05 & $1.42 \mathrm{E}-07$ & $2.66 \mathrm{E}-06$ & 0.942 & 0.916 & 0.968 & 0.834 & 0.853 & 0.813 \\
\hline hsa-miR-130a-3p_477851_mir & -2.62 & $1.85 \mathrm{E}-06$ & $2.00 \mathrm{E}-05$ & 0.94 & 0.909 & 0.972 & 0.861 & 0.884 & 0.835 \\
\hline hsa-miR-214-3p_477974_mir & -7.59 & $1.19 \mathrm{E}-07$ & $2.49 \mathrm{E}-06$ & 0.937 & 0.901 & 0.972 & 0.896 & 0.94 & 0.846 \\
\hline hsa-miR-10a-5p_479241_mir & -2.19 & $1.44 \mathrm{E}-02$ & $2.82 \mathrm{E}-02$ & 0.937 & 0.906 & 0.969 & 0.897 & 0.904 & 0.889 \\
\hline hsa-miR-497-5p_478138_mir & -3.68 & $2.13 \mathrm{E}-04$ & $8.08 \mathrm{E}-04$ & 0.936 & 0.911 & 0.961 & 0.814 & 0.829 & 0.797 \\
\hline hsa-miR-143-3p_477912_mir & -3.15 & $1.58 \mathrm{E}-06$ & $1.81 \mathrm{E}-05$ & 0.936 & 0.906 & 0.965 & 0.86 & 0.868 & 0.85 \\
\hline hsa-miR-20a-5p_478586_mir & -2.79 & $5.14 \mathrm{E}-08$ & $1.30 E-06$ & 0.933 & 0.901 & 0.964 & 0.877 & 0.906 & 0.843 \\
\hline hsa-miR-29c-3p_479229_mir & -3.55 & $2.14 \mathrm{E}-04$ & $8.08 \mathrm{E}-04$ & 0.931 & 0.897 & 0.965 & 0.86 & 0.84 & 0.883 \\
\hline hsa-miR-17-5p_478447_mir & -3.68 & 4.83E-05 & $2.41 \mathrm{E}-04$ & 0.93 & 0.893 & 0.966 & 0.874 & 0.898 & 0.847 \\
\hline hsa-miR-486-5p_478128_mir & -11.10 & $2.21 \mathrm{E}-07$ & $3.73 \mathrm{E}-06$ & 0.929 & 0.899 & 0.958 & 0.853 & 0.818 & 0.893 \\
\hline hsa-miR-145-5p_477916_mir & -3.07 & $6.47 \mathrm{E}-06$ & $5.34 \mathrm{E}-05$ & 0.929 & 0.899 & 0.958 & 0.877 & 0.901 & 0.851 \\
\hline hsa-miR-214-5p_478768_mir & -8.13 & $2.33 \mathrm{E}-08$ & $9.85 \mathrm{E}-07$ & 0.923 & 0.885 & 0.96 & 0.877 & 0.906 & 0.844 \\
\hline hsa-miR-20b-5p_477804_mir & -11.65 & $3.73 \mathrm{E}-09$ & $2.65 \mathrm{E}-07$ & 0.921 & 0.887 & 0.956 & 0.883 & 0.879 & 0.887 \\
\hline hsa-miR-551b-3p_478159_mir & -9.71 & $2.07 \mathrm{E}-10$ & $3.68 \mathrm{E}-08$ & 0.919 & 0.885 & 0.953 & 0.852 & 0.906 & 0.79 \\
\hline hsa-miR-107_478254_mir & -4.70 & $1.35 \mathrm{E}-03$ & $3.73 \mathrm{E}-03$ & 0.917 & 0.883 & 0.951 & 0.919 & 0.938 & 0.898 \\
\hline hsa-miR-202-5p_478755_mir & -7.19 & $5.11 \mathrm{E}-08$ & $1.30 \mathrm{E}-06$ & 0.915 & 0.876 & 0.954 & 0.855 & 0.867 & 0.842 \\
\hline hsa-miR-93-5p_478210_mir & -2.84 & $3.88 \mathrm{E}-04$ & $1.30 \mathrm{E}-03$ & 0.915 & 0.875 & 0.954 & 0.86 & 0.872 & 0.847 \\
\hline hsa-miR-483-3p_478122_mir & -7.01 & $3.69 E-09$ & $2.65 \mathrm{E}-07$ & 0.913 & 0.877 & 0.949 & 0.837 & 0.884 & 0.784 \\
\hline hsa-miR-652-3p_478189_mir & -2.05 & $2.63 \mathrm{E}-05$ & $1.70 \mathrm{E}-04$ & 0.913 & 0.882 & 0.945 & 0.825 & 0.831 & 0.818 \\
\hline hsa-miR-29b-3p_478369_mir & -3.59 & $3.71 \mathrm{E}-06$ & $3.47 \mathrm{E}-05$ & 0.911 & 0.877 & 0.945 & 0.836 & 0.834 & 0.838 \\
\hline hsa-miR-328-3p_478028_mir & -5.03 & $2.86 \mathrm{E}-04$ & $1.01 \mathrm{E}-03$ & 0.908 & 0.874 & 0.941 & 0.819 & 0.798 & 0.842 \\
\hline hsa-miR-25-3p_477994_mir & -2.37 & $3.44 \mathrm{E}-05$ & $1.99 \mathrm{E}-04$ & 0.908 & 0.87 & 0.946 & 0.865 & 0.899 & 0.827 \\
\hline hsa-miR-26a-5p_477995_mir & -2.30 & $1.99 \mathrm{E}-05$ & $1.38 \mathrm{E}-04$ & 0.904 & 0.872 & 0.937 & 0.796 & 0.799 & 0.791 \\
\hline hsa-miR-296-5p_477836_mir & -6.51 & $3.42 \mathrm{E}-05$ & $1.99 \mathrm{E}-04$ & 0.903 & 0.865 & 0.941 & 0.878 & 0.796 & 0.973 \\
\hline hsa-miR-144-3p_477913_mir & -5.05 & $3.53 \mathrm{E}-05$ & $1.99 \mathrm{E}-04$ & 0.903 & 0.867 & 0.938 & 0.827 & 0.85 & 0.801 \\
\hline hsa-miR-769-5p_478203_mir & -3.98 & $3.53 \mathrm{E}-05$ & 1.99E-04 & 0.903 & 0.864 & 0.942 & 0.899 & 0.901 & 0.896 \\
\hline hsa-miR-181a-5p_477857_mir & -2.13 & $5.21 \mathrm{E}-06$ & $4.52 \mathrm{E}-05$ & 0.902 & 0.865 & 0.939 & 0.843 & 0.895 & 0.783 \\
\hline hsa-miR-29a-3p_478587_mir & -3.12 & $1.16 \mathrm{E}-03$ & $3.38 \mathrm{E}-03$ & 0.901 & 0.86 & 0.942 & 0.878 & 0.854 & 0.905 \\
\hline hsa-miR-152-3p_477921_mir & -3.98 & $2.17 \mathrm{E}-05$ & $1.48 \mathrm{E}-04$ & 0.9 & 0.866 & 0.934 & 0.804 & 0.791 & 0.819 \\
\hline hsa-miR-125b-1-3p_478665_mir & -9.13 & $1.93 \mathrm{E}-07$ & $3.42 \mathrm{E}-06$ & 0.895 & 0.86 & 0.931 & 0.86 & 0.868 & 0.851 \\
\hline hsa-miR-30a-3p_478273_mir & -1.66 & $9.38 \mathrm{E}-05$ & 4.27E-04 & 0.891 & 0.854 & 0.928 & 0.812 & 0.793 & 0.834 \\
\hline hsa-miR-449b-5p_479528_mir & -10.10 & $2.30 \mathrm{E}-08$ & $9.85 \mathrm{E}-07$ & 0.889 & 0.846 & 0.932 & 0.88 & 0.908 & 0.847 \\
\hline hsa-miR-219a-5p_477980_mir & -6.58 & $5.10 \mathrm{E}-07$ & 7.54E-06 & 0.889 & 0.852 & 0.926 & 0.84 & 0.854 & 0.824 \\
\hline hsa-miR-125a-5p_477884_mir & -1.40 & $1.48 \mathrm{E}-03$ & $4.02 E-03$ & 0.888 & 0.846 & 0.93 & 0.827 & 0.84 & 0.813 \\
\hline
\end{tabular}


Table 2 (continued)

\begin{tabular}{|c|c|c|c|c|c|c|c|c|c|}
\hline miRNA & LogFC & p-value & Adj. p-value & AUC & $\begin{array}{l}\text { AUC 95\% } \\
\text { Cl_lower }\end{array}$ & $\begin{array}{l}\text { AUC 95\% } \\
\text { Cl_upper }\end{array}$ & Accuracy & Sensitivity & Specificity \\
\hline hsa-miR-374b-3p_479421_mir & -4.64 & $2.15 \mathrm{E}-06$ & $2.24 \mathrm{E}-05$ & 0.887 & 0.845 & 0.929 & 0.8 & 0.791 & 0.81 \\
\hline hsa-miR-101-3p_477863_mir & -4.19 & $6.98 \mathrm{E}-05$ & $3.39 \mathrm{E}-04$ & 0.886 & 0.845 & 0.927 & 0.832 & 0.896 & 0.759 \\
\hline hsa-miR-452-5p_478109_mir & -3.88 & $2.89 \mathrm{E}-05$ & $1.80 \mathrm{E}-04$ & 0.886 & 0.852 & 0.92 & 0.751 & 0.737 & 0.766 \\
\hline hsa-miR-193a-3p_478306_mir & -2.46 & 2.37E-03 & 5.97E-03 & 0.884 & 0.841 & 0.928 & 0.869 & 0.899 & 0.835 \\
\hline hsa-miR-148a-3p_477814_mir & -1.48 & $1.24 \mathrm{E}-03$ & $3.52 \mathrm{E}-03$ & 0.884 & 0.845 & 0.923 & 0.822 & 0.818 & 0.826 \\
\hline hsa-miR-133a-3p_478511_mir & -6.89 & $1.67 \mathrm{E}-04$ & $6.73 \mathrm{E}-04$ & 0.883 & 0.843 & 0.922 & 0.827 & 0.853 & 0.797 \\
\hline hsa-miR-675-5p_478196_mir & -4.22 & $5.46 \mathrm{E}-06$ & 4.61E-05 & 0.883 & 0.845 & 0.92 & 0.766 & 0.721 & 0.817 \\
\hline hsa-miR-34a-5p_478048_mir & -2.04 & $3.41 \mathrm{E}-05$ & $1.99 \mathrm{E}-04$ & 0.882 & 0.841 & 0.922 & 0.823 & 0.801 & 0.848 \\
\hline hsa-miR-582-5p_478166_mir & -8.33 & $3.37 \mathrm{E}-07$ & $5.20 \mathrm{E}-06$ & 0.881 & 0.842 & 0.92 & 0.84 & 0.767 & 0.923 \\
\hline hsa-miR-2110_477971_mir & -4.46 & $2.45 \mathrm{E}-06$ & $2.48 \mathrm{E}-05$ & 0.879 & 0.837 & 0.92 & 0.782 & 0.794 & 0.769 \\
\hline hsa-miR-185-5p_477939_mir & -2.69 & $1.09 \mathrm{E}-02$ & $2.18 \mathrm{E}-02$ & 0.879 & 0.836 & 0.921 & 0.828 & 0.872 & 0.777 \\
\hline hsa-miR-144-5p_477914_mir & -11.46 & 4.47E-08 & $1.30 \mathrm{E}-06$ & 0.877 & 0.833 & 0.921 & 0.873 & 0.944 & 0.791 \\
\hline hsa-miR-199a-5p_478231_mir & -7.83 & $1.24 \mathrm{E}-04$ & $5.31 \mathrm{E}-04$ & 0.877 & 0.831 & 0.923 & 0.772 & 0.712 & 0.84 \\
\hline hsa-miR-361-5p_478056_mir & -1.48 & $9.56 \mathrm{E}-04$ & $2.83 \mathrm{E}-03$ & 0.877 & 0.837 & 0.918 & 0.821 & 0.86 & 0.778 \\
\hline hsa-miR-195-5p_477957_mir & -2.80 & $2.19 \mathrm{E}-04$ & $8.17 \mathrm{E}-04$ & 0.875 & 0.832 & 0.919 & 0.835 & 0.822 & 0.849 \\
\hline hsa-miR-136-5p_478307_mir & -8.01 & $5.88 \mathrm{E}-07$ & 8.03E-06 & 0.873 & 0.834 & 0.913 & 0.828 & 0.821 & 0.836 \\
\hline hsa-miR-548d-5p_480870_mir & -3.80 & $3.79 \mathrm{E}-03$ & 8.96E-03 & 0.873 & 0.827 & 0.919 & 0.755 & 0.65 & 0.875 \\
\hline hsa-miR-30b-5p_478007_mir & -2.55 & $2.64 \mathrm{E}-03$ & $6.47 \mathrm{E}-03$ & 0.873 & 0.829 & 0.916 & 0.774 & 0.795 & 0.751 \\
\hline hsa-miR-363-3p_478060_mir & -8.98 & $3.58 \mathrm{E}-05$ & $1.99 \mathrm{E}-04$ & 0.869 & 0.825 & 0.914 & 0.782 & 0.776 & 0.789 \\
\hline hsa-miR-27b-3p_478270_mir & -2.46 & 2.87E-02 & 4.90E-02 & 0.868 & 0.828 & 0.908 & 0.76 & 0.815 & 0.698 \\
\hline hsa-miR-24-3p_477992_mir & -2.06 & $4.68 \mathrm{E}-05$ & $2.40 E-04$ & 0.868 & 0.823 & 0.913 & 0.859 & 0.9 & 0.813 \\
\hline hsa-miR-499a-5p_478139_mir & -4.05 & $4.42 \mathrm{E}-05$ & $2.34 \mathrm{E}-04$ & 0.864 & 0.821 & 0.908 & 0.851 & 0.875 & 0.824 \\
\hline hsa-miR-15a-5p_477858_mir & -1.96 & $3.80 \mathrm{E}-04$ & $1.28 \mathrm{E}-03$ & 0.863 & 0.823 & 0.903 & 0.77 & 0.781 & 0.758 \\
\hline hsa-miR-31-3p_478012_mir & -5.27 & $2.92 \mathrm{E}-04$ & $1.02 \mathrm{E}-03$ & 0.86 & 0.817 & 0.904 & 0.799 & 0.766 & 0.836 \\
\hline hsa-miR-18a-3p_477944_mir & -4.70 & $5.55 \mathrm{E}-05$ & $2.74 \mathrm{E}-04$ & 0.859 & 0.817 & 0.901 & 0.761 & 0.747 & 0.777 \\
\hline hsa-miR-92a-3p_477827_mir & -1.48 & $5.48 \mathrm{E}-04$ & $1.74 \mathrm{E}-03$ & 0.859 & 0.811 & 0.907 & 0.813 & 0.822 & 0.804 \\
\hline hsa-miR-130b-3p_477840_mir & -5.57 & $3.72 \mathrm{E}-04$ & $1.27 \mathrm{E}-03$ & 0.858 & 0.815 & 0.901 & 0.748 & 0.644 & 0.866 \\
\hline hsa-let-7b-5p_478576_mir & -1.57 & $5.76 \mathrm{E}-04$ & $1.81 \mathrm{E}-03$ & 0.858 & 0.814 & 0.902 & 0.76 & 0.758 & 0.761 \\
\hline hsa-miR-30e-3p_478388_mir & -4.70 & $5.51 \mathrm{E}-03$ & $1.22 \mathrm{E}-02$ & 0.854 & 0.81 & 0.899 & 0.753 & 0.628 & 0.897 \\
\hline hsa-miR-23b-5p_477991_mir & -3.62 & $3.36 \mathrm{E}-05$ & $1.99 \mathrm{E}-04$ & 0.853 & 0.81 & 0.896 & 0.756 & 0.758 & 0.754 \\
\hline hsa-miR-29b-2-5p_478003_mir & -4.33 & $2.79 \mathrm{E}-06$ & $2.75 \mathrm{E}-05$ & 0.85 & 0.806 & 0.894 & 0.797 & 0.806 & 0.786 \\
\hline hsa-miR-30e-5p_479235_mir & -8.36 & $6.16 \mathrm{E}-04$ & $1.92 \mathrm{E}-03$ & 0.849 & 0.802 & 0.896 & 0.709 & 0.638 & 0.791 \\
\hline hsa-miR-200c-3p_478351_mir & -7.14 & $2.32 \mathrm{E}-05$ & $1.53 \mathrm{E}-04$ & 0.848 & 0.802 & 0.894 & 0.802 & 0.721 & 0.894 \\
\hline hsa-miR-1180-3p_477869_mir & -3.91 & $4.43 \mathrm{E}-05$ & $2.34 \mathrm{E}-04$ & 0.847 & 0.798 & 0.896 & 0.806 & 0.754 & 0.866 \\
\hline hsa-miR-190a-5p_478358_mir & -1.89 & $1.87 \mathrm{E}-02$ & $3.48 \mathrm{E}-02$ & 0.847 & 0.801 & 0.892 & 0.829 & 0.814 & 0.847 \\
\hline hsa-miR-151b_477811_mir & -9.82 & $2.25 \mathrm{E}-04$ & $8.31 \mathrm{E}-04$ & 0.846 & 0.801 & 0.892 & 0.761 & 0.733 & 0.792 \\
\hline hsa-miR-505-5p_478957_mir & -5.86 & 4.24E-05 & $2.32 \mathrm{E}-04$ & 0.846 & 0.801 & 0.891 & 0.796 & 0.805 & 0.786 \\
\hline hsa-miR-196b-5p_478585_mir & -6.46 & $1.86 \mathrm{E}-06$ & $2.00 E-05$ & 0.845 & 0.795 & 0.894 & 0.767 & 0.67 & 0.879 \\
\hline hsa-miR-324-5p_478024_mir & -1.51 & $8.13 \mathrm{E}-03$ & 1.65E-02 & 0.843 & 0.797 & 0.889 & 0.777 & 0.754 & 0.802 \\
\hline hsa-miR-224-5p_477986_mir & -2.72 & $1.58 \mathrm{E}-04$ & $6.45 \mathrm{E}-04$ & 0.842 & 0.8 & 0.883 & 0.746 & 0.695 & 0.804 \\
\hline hsa-miR-139-5p_478312_mir & -5.12 & $2.43 \mathrm{E}-04$ & $8.80 \mathrm{E}-04$ & 0.839 & 0.794 & 0.885 & 0.727 & 0.725 & 0.729 \\
\hline hsa-miR-545-5p_479003_mir & -5.30 & $8.42 \mathrm{E}-06$ & $6.79 E-05$ & 0.838 & 0.79 & 0.886 & 0.79 & 0.79 & 0.79 \\
\hline hsa-miR-222-3p_477982_mir & -2.08 & $7.82 \mathrm{E}-04$ & $2.37 \mathrm{E}-03$ & 0.838 & 0.791 & 0.884 & 0.728 & 0.762 & 0.689 \\
\hline hsa-miR-340-5p_478042_mir & -9.05 & $9.31 \mathrm{E}-06$ & $7.18 \mathrm{E}-05$ & 0.836 & 0.79 & 0.882 & 0.803 & 0.817 & 0.786 \\
\hline hsa-miR-504-5p_478144_mir & -3.54 & $1.86 \mathrm{E}-04$ & $7.18 \mathrm{E}-04$ & 0.836 & 0.787 & 0.886 & 0.778 & 0.729 & 0.833 \\
\hline hsa-miR-106a-5p_478225_mir & -10.76 & $4.30 \mathrm{E}-08$ & $1.30 \mathrm{E}-06$ & 0.834 & 0.784 & 0.883 & 0.816 & 0.924 & 0.693 \\
\hline hsa-miR-1271-5p_478674_mir & -9.10 & $3.87 \mathrm{E}-06$ & $3.52 \mathrm{E}-05$ & 0.834 & 0.785 & 0.883 & 0.823 & 0.9 & 0.734 \\
\hline hsa-miR-125b-2-3p_478666_mir & -6.80 & $9.11 \mathrm{E}-06$ & $7.18 \mathrm{E}-05$ & 0.834 & 0.782 & 0.886 & 0.804 & 0.866 & 0.735 \\
\hline hsa-miR-339-3p_478325_mir & -3.60 & $6.56 \mathrm{E}-03$ & $1.39 \mathrm{E}-02$ & 0.834 & 0.782 & 0.886 & 0.778 & 0.692 & 0.876 \\
\hline
\end{tabular}


Table 2 (continued)

\begin{tabular}{|c|c|c|c|c|c|c|c|c|c|}
\hline miRNA & LogFC & p-value & Adj. p-value & AUC & $\begin{array}{l}\text { AUC 95\% } \\
\text { Cl_lower }\end{array}$ & $\begin{array}{l}\text { AUC 95\% } \\
\text { Cl_upper }\end{array}$ & Accuracy & Sensitivity & Specificity \\
\hline hsa-miR-483-5p_478432_mir & -6.45 & $5.42 \mathrm{E}-07$ & $7.69 \mathrm{E}-06$ & 0.832 & 0.783 & 0.882 & 0.785 & 0.871 & 0.687 \\
\hline hsa-miR-584-5p_478167_mir & -10.36 & $6.40 \mathrm{E}-07$ & $8.25 \mathrm{E}-06$ & 0.831 & 0.781 & 0.882 & 0.803 & 0.906 & 0.685 \\
\hline hsa-miR-17-3p_477932_mir & -8.43 & $2.22 \mathrm{E}-05$ & $1.49 \mathrm{E}-04$ & 0.831 & 0.779 & 0.883 & 0.805 & 0.861 & 0.741 \\
\hline hsa-miR-570-3p_479053_mir & -4.46 & $1.11 \mathrm{E}-04$ & $4.79 E-04$ & 0.831 & 0.785 & 0.876 & 0.766 & 0.697 & 0.844 \\
\hline hsa-miR-625-5p_479469_mir & -10.93 & $4.36 \mathrm{E}-06$ & $3.87 \mathrm{E}-05$ & 0.83 & 0.781 & 0.88 & 0.806 & 0.865 & 0.738 \\
\hline hsa-miR-196a-5p_478230_mir & -7.37 & $1.01 \mathrm{E}-05$ & $7.64 \mathrm{E}-05$ & 0.83 & 0.777 & 0.883 & 0.812 & 0.879 & 0.735 \\
\hline hsa-miR-7-1-3p_478198_mir & -7.37 & $1.00 \mathrm{E}-04$ & $4.52 \mathrm{E}-04$ & 0.829 & 0.783 & 0.874 & 0.762 & 0.646 & 0.895 \\
\hline hsa-miR-450b-5p_478914_mir & -9.65 & $1.03 \mathrm{E}-07$ & $2.29 \mathrm{E}-06$ & 0.828 & 0.775 & 0.88 & 0.803 & 0.906 & 0.685 \\
\hline hsa-miR-221-5p_478778_mir & -5.01 & $3.08 \mathrm{E}-04$ & $1.06 \mathrm{E}-03$ & 0.827 & 0.779 & 0.875 & 0.745 & 0.64 & 0.865 \\
\hline hsa-miR-128-3p_477892_mir & -1.32 & $5.21 \mathrm{E}-03$ & $1.16 \mathrm{E}-02$ & 0.823 & 0.772 & 0.874 & 0.743 & 0.741 & 0.746 \\
\hline hsa-miR-491-5p_478132_mir & -3.50 & $1.88 \mathrm{E}-03$ & $4.92 \mathrm{E}-03$ & 0.822 & 0.774 & 0.87 & 0.743 & 0.703 & 0.79 \\
\hline hsa-miR-136-3p_477902_mir & -7.94 & $2.78 \mathrm{E}-05$ & $1.76 \mathrm{E}-04$ & 0.821 & 0.771 & 0.871 & 0.786 & 0.855 & 0.707 \\
\hline hsa-miR-101-5p_478620_mir & -7.44 & $1.09 \mathrm{E}-05$ & $8.08 \mathrm{E}-05$ & 0.819 & 0.766 & 0.873 & 0.812 & 0.877 & 0.738 \\
\hline hsa-miR-151a-3p_477919_mir & -1.93 & $2.85 \mathrm{E}-04$ & $1.01 \mathrm{E}-03$ & 0.819 & 0.769 & 0.87 & 0.803 & 0.854 & 0.745 \\
\hline hsa-miR-28-3p_477999_mir & -2.25 & $4.30 \mathrm{E}-03$ & $1.00 \mathrm{E}-02$ & 0.817 & 0.771 & 0.863 & 0.738 & 0.728 & 0.749 \\
\hline hsa-miR-489-3p_478130_mir & -4.40 & $1.33 \mathrm{E}-04$ & $5.62 \mathrm{E}-04$ & 0.815 & 0.766 & 0.865 & 0.76 & 0.736 & 0.787 \\
\hline hsa-miR-106b-3p_477866_mir & -2.41 & $6.48 \mathrm{E}-03$ & $1.39 \mathrm{E}-02$ & 0.815 & 0.765 & 0.866 & 0.72 & 0.693 & 0.751 \\
\hline hsa-miR-324-3p_478023_mir & -7.87 & $2.85 E-04$ & $1.01 \mathrm{E}-03$ & 0.814 & 0.766 & 0.861 & 0.761 & 0.686 & 0.847 \\
\hline hsa-miR-125a-3p_477883_mir & -3.24 & $4.18 \mathrm{E}-04$ & $1.39 \mathrm{E}-03$ & 0.811 & 0.76 & 0.862 & 0.765 & 0.741 & 0.791 \\
\hline hsa-let-7i-3p_477862_mir & -6.66 & $4.69 \mathrm{E}-04$ & $1.54 \mathrm{E}-03$ & 0.81 & 0.759 & 0.861 & 0.747 & 0.665 & 0.841 \\
\hline hsa-miR-33b-5p_478479_mir & -5.49 & $2.06 \mathrm{E}-03$ & $5.30 E-03$ & 0.81 & 0.758 & 0.862 & 0.704 & 0.626 & 0.793 \\
\hline hsa-miR-503-5p_478143_mir & -2.80 & $6.04 \mathrm{E}-03$ & $1.31 \mathrm{E}-02$ & 0.81 & 0.757 & 0.863 & 0.762 & 0.726 & 0.805 \\
\hline hsa-miR-301a-3p_477815_mir & -5.08 & $1.51 \mathrm{E}-03$ & 4.05E-03 & 0.809 & 0.76 & 0.858 & 0.723 & 0.608 & 0.856 \\
\hline hsa-miR-330-3p_478030_mir & -5.72 & $7.79 \mathrm{E}-04$ & $2.37 \mathrm{E}-03$ & 0.805 & 0.754 & 0.856 & 0.761 & 0.645 & 0.892 \\
\hline hsa-miR-425-5p_478094_mir & -1.51 & $2.66 \mathrm{E}-02$ & 4.63E-02 & 0.805 & 0.757 & 0.852 & 0.728 & 0.714 & 0.744 \\
\hline hsa-miR-16-2-3p_477931_mir & -3.32 & $1.12 \mathrm{E}-02$ & $2.22 \mathrm{E}-02$ & 0.804 & 0.747 & 0.862 & 0.78 & 0.769 & 0.793 \\
\hline hsa-miR-548k_479374_mir & -14.17 & $8.73 \mathrm{E}-03$ & $1.76 \mathrm{E}-02$ & 0.801 & 0.757 & 0.845 & 0.718 & 0.654 & 0.79 \\
\hline hsa-miR-429_477849_mir & -2.00 & $1.76 \mathrm{E}-02$ & $3.37 \mathrm{E}-02$ & 0.801 & 0.747 & 0.854 & 0.768 & 0.807 & 0.723 \\
\hline hsa-miR-598-3p_478172_mir & -1.51 & $4.51 \mathrm{E}-03$ & $1.04 \mathrm{E}-02$ & 0.8 & 0.754 & 0.847 & 0.696 & 0.726 & 0.661 \\
\hline hsa-miR-887-3p_479189_mir & -5.41 & $1.42 \mathrm{E}-04$ & $5.95 \mathrm{E}-04$ & 0.799 & 0.748 & 0.85 & 0.737 & 0.632 & 0.858 \\
\hline hsa-miR-93-3p_478209_mir & -4.60 & $2.28 \mathrm{E}-04$ & $8.33 \mathrm{E}-04$ & 0.798 & 0.745 & 0.852 & 0.749 & 0.702 & 0.801 \\
\hline hsa-miR-629-5p_478183_mir & -6.16 & $1.75 \mathrm{E}-04$ & $6.90 \mathrm{E}-04$ & 0.796 & 0.746 & 0.846 & 0.753 & 0.678 & 0.839 \\
\hline hsa-miR-21-5p_477975_mir & 1.32 & $7.91 \mathrm{E}-03$ & $1.61 \mathrm{E}-02$ & 0.796 & 0.751 & 0.842 & 0.683 & 0.688 & 0.677 \\
\hline hsa-miR-140-3p_477908_mir & -3.42 & $6.10 \mathrm{E}-03$ & $1.31 \mathrm{E}-02$ & 0.793 & 0.743 & 0.843 & 0.734 & 0.752 & 0.713 \\
\hline hsa-miR-425-3p_478093_mir & -5.28 & $9.90 E-04$ & $2.90 \mathrm{E}-03$ & 0.792 & 0.74 & 0.844 & 0.728 & 0.588 & 0.888 \\
\hline hsa-miR-200a-5p_478752_mir & 3.05 & $2.34 \mathrm{E}-02$ & 4.15E-02 & 0.792 & 0.735 & 0.85 & 0.713 & 0.693 & 0.736 \\
\hline hsa-miR-590-3p_478168_mir & -4.77 & 1.27E-03 & $3.56 \mathrm{E}-03$ & 0.791 & 0.739 & 0.842 & 0.702 & 0.637 & 0.776 \\
\hline hsa-miR-30a-5p_479448_mir & -8.52 & $7.71 \mathrm{E}-04$ & $2.37 \mathrm{E}-03$ & 0.789 & 0.738 & 0.841 & 0.734 & 0.726 & 0.744 \\
\hline hsa-let-7 g-3p_477850_mir & -6.38 & $1.74 \mathrm{E}-04$ & $6.90 \mathrm{E}-04$ & 0.787 & 0.733 & 0.84 & 0.756 & 0.608 & 0.926 \\
\hline hsa-miR-542-3p_478153_mir & -8.15 & $2.88 \mathrm{E}-06$ & $2.76 \mathrm{E}-05$ & 0.786 & 0.729 & 0.842 & 0.778 & 0.901 & 0.638 \\
\hline hsa-miR-31-5p_478015_mir & -1.78 & $7.34 \mathrm{E}-03$ & $1.51 \mathrm{E}-02$ & 0.786 & 0.737 & 0.835 & 0.676 & 0.708 & 0.64 \\
\hline hsa-miR-379-5p_478077_mir & -5.15 & $5.46 \mathrm{E}-04$ & $1.74 \mathrm{E}-03$ & 0.78 & 0.724 & 0.835 & 0.755 & 0.773 & 0.734 \\
\hline hsa-miR-194-5p_477956_mir & -1.66 & $1.21 \mathrm{E}-03$ & $3.48 \mathrm{E}-03$ & 0.78 & 0.724 & 0.836 & 0.778 & 0.861 & 0.683 \\
\hline hsa-miR-34c-5p_478052_mir & -2.51 & 4.47E-03 & $1.04 \mathrm{E}-02$ & 0.779 & 0.726 & 0.832 & 0.685 & 0.639 & 0.738 \\
\hline hsa-miR-576-5p_478165_mir & -6.85 & $8.70 E-05$ & 4.01E-04 & 0.778 & 0.721 & 0.836 & 0.774 & 0.853 & 0.684 \\
\hline hsa-miR-28-5p_478000_mir & -5.87 & $1.45 \mathrm{E}-03$ & $3.96 \mathrm{E}-03$ & 0.778 & 0.726 & 0.829 & 0.731 & 0.64 & 0.835 \\
\hline hsa-miR-708-3p_479162_mir & -2.77 & $2.27 \mathrm{E}-03$ & $5.75 E-03$ & 0.772 & 0.715 & 0.828 & 0.738 & 0.74 & 0.736 \\
\hline hsa-miR-505-3p_478145_mir & -2.12 & $3.62 \mathrm{E}-03$ & 8.67E-03 & 0.771 & 0.718 & 0.824 & 0.758 & 0.737 & 0.781 \\
\hline hsa-miR-26b-5p_478418_mir & -1.01 & $2.37 \mathrm{E}-02$ & 4.19E-02 & 0.768 & 0.717 & 0.819 & 0.692 & 0.714 & 0.666 \\
\hline
\end{tabular}


Table 2 (continued)

\begin{tabular}{|c|c|c|c|c|c|c|c|c|c|}
\hline miRNA & LogFC & p-value & Adj. p-value & AUC & $\begin{array}{l}\text { AUC 95\% } \\
\text { Cl_lower }\end{array}$ & $\begin{array}{l}\text { AUC 95\% } \\
\text { Cl_upper }\end{array}$ & Accuracy & Sensitivity & Specificity \\
\hline hsa-miR-365a-3p_478065_mir & -4.75 & $2.67 \mathrm{E}-02$ & $4.63 \mathrm{E}-02$ & 0.767 & 0.713 & 0.822 & 0.661 & 0.495 & 0.85 \\
\hline hsa-miR-423-3p_478327_mir & -1.63 & $1.58 \mathrm{E}-03$ & $4.19 \mathrm{E}-03$ & 0.765 & 0.716 & 0.815 & 0.65 & 0.687 & 0.607 \\
\hline hsa-miR-338-5p_478038_mir & -3.61 & $2.40 \mathrm{E}-03$ & $6.01 \mathrm{E}-03$ & 0.761 & 0.703 & 0.819 & 0.704 & 0.719 & 0.687 \\
\hline hsa-miR-210-3p_477970_mir & -1.17 & $1.67 \mathrm{E}-02$ & $3.22 \mathrm{E}-02$ & 0.761 & 0.708 & 0.815 & 0.672 & 0.698 & 0.643 \\
\hline hsa-miR-551a_478158_mir & -5.58 & 1.97E-03 & $5.10 \mathrm{E}-03$ & 0.76 & 0.699 & 0.822 & 0.741 & 0.69 & 0.8 \\
\hline hsa-miR-889-3p_478208_mir & -8.68 & $1.14 \mathrm{E}-05$ & $8.28 \mathrm{E}-05$ & 0.759 & 0.698 & 0.82 & 0.789 & 0.922 & 0.637 \\
\hline hsa-miR-301b-3p_477825_mir & -5.75 & $1.87 \mathrm{E}-02$ & $3.48 \mathrm{E}-02$ & 0.758 & 0.7 & 0.816 & 0.751 & 0.851 & 0.637 \\
\hline hsa-miR-590-5p_478367_mir & -3.83 & $1.63 \mathrm{E}-02$ & $3.15 \mathrm{E}-02$ & 0.757 & 0.703 & 0.812 & 0.707 & 0.59 & 0.841 \\
\hline hsa-miR-548am-5p_480872_mir & -3.25 & $1.13 \mathrm{E}-02$ & $2.22 \mathrm{E}-02$ & 0.757 & 0.703 & 0.81 & 0.663 & 0.526 & 0.82 \\
\hline hsa-miR-187-3p_477941_mir & -4.47 & $6.97 \mathrm{E}-03$ & $1.46 \mathrm{E}-02$ & 0.754 & 0.701 & 0.807 & 0.704 & 0.644 & 0.772 \\
\hline hsa-miR-450a-5p_478106_mir & -7.12 & $1.26 \mathrm{E}-03$ & $3.56 \mathrm{E}-03$ & 0.753 & 0.692 & 0.814 & 0.743 & 0.646 & 0.853 \\
\hline hsa-miR-376a-5p_478859_mir & -5.94 & $1.04 \mathrm{E}-04$ & $4.56 \mathrm{E}-04$ & 0.75 & 0.688 & 0.811 & 0.738 & 0.832 & 0.631 \\
\hline hsa-miR-1296-5p_479451_mir & -4.26 & $2.49 \mathrm{E}-03$ & $6.17 \mathrm{E}-03$ & 0.75 & 0.695 & 0.806 & 0.703 & 0.719 & 0.686 \\
\hline hsa-miR-181c-3p_477933_mir & -3.64 & $9.29 \mathrm{E}-04$ & 2.77E-03 & 0.748 & 0.685 & 0.811 & 0.715 & 0.805 & 0.613 \\
\hline hsa-miR-1247-5p_477882_mir & -3.36 & $2.43 \mathrm{E}-02$ & 4.26E-02 & 0.748 & 0.692 & 0.804 & 0.698 & 0.586 & 0.827 \\
\hline hsa-miR-34a-3p_478047_mir & -2.32 & $1.77 \mathrm{E}-02$ & $3.37 \mathrm{E}-02$ & 0.748 & 0.694 & 0.803 & 0.658 & 0.727 & 0.579 \\
\hline hsa-miR-654-3p_479135_mir & -7.56 & 4.77E-04 & $1.55 \mathrm{E}-03$ & 0.74 & 0.681 & 0.799 & 0.76 & 0.692 & 0.839 \\
\hline hsa-miR-411-5p_478086_mir & -5.12 & 7.16E-03 & $1.49 \mathrm{E}-02$ & 0.738 & 0.68 & 0.796 & 0.715 & 0.647 & 0.793 \\
\hline hsa-miR-181d-5p_479517_mir & -5.67 & $4.10 \mathrm{E}-03$ & 9.63E-03 & 0.733 & 0.675 & 0.791 & 0.686 & 0.554 & 0.838 \\
\hline hsa-miR-200b-3p_477963_mir & -3.43 & $7.26 \mathrm{E}-03$ & $1.50 E-02$ & 0.733 & 0.674 & 0.792 & 0.683 & 0.609 & 0.767 \\
\hline hsa-miR-299-3p_478792_mir & -6.51 & 4.73E-05 & $2.40 E-04$ & 0.732 & 0.67 & 0.794 & 0.748 & 0.893 & 0.582 \\
\hline hsa-miR-182-5p_477935_mir & -8.68 & 8.37E-05 & $3.91 \mathrm{E}-04$ & 0.73 & 0.666 & 0.795 & 0.753 & 0.9 & 0.585 \\
\hline hsa-miR-410-3p_478085_mir & -5.65 & $1.22 \mathrm{E}-03$ & $3.48 \mathrm{E}-03$ & 0.728 & 0.67 & 0.787 & 0.753 & 0.771 & 0.733 \\
\hline hsa-miR-744-5p_478200_mir & -3.55 & $1.81 \mathrm{E}-02$ & $3.40 \mathrm{E}-02$ & 0.725 & 0.67 & 0.781 & 0.657 & 0.637 & 0.68 \\
\hline hsa-miR-96-5p_478215_mir & -6.84 & $1.03 \mathrm{E}-04$ & $4.56 \mathrm{E}-04$ & 0.724 & 0.658 & 0.79 & 0.744 & 0.886 & 0.583 \\
\hline hsa-miR-133b_480871_mir & -3.18 & $1.98 \mathrm{E}-02$ & $3.66 \mathrm{E}-02$ & 0.724 & 0.664 & 0.783 & 0.686 & 0.651 & 0.726 \\
\hline hsa-miR-544a_478156_mir & -5.67 & 1.37E-03 & $3.77 \mathrm{E}-03$ & 0.719 & 0.66 & 0.779 & 0.756 & 0.81 & 0.694 \\
\hline hsa-miR-497-3p_478946_mir & -6.92 & $1.44 \mathrm{E}-04$ & $5.95 \mathrm{E}-04$ & 0.718 & 0.652 & 0.784 & 0.753 & 0.899 & 0.585 \\
\hline hsa-miR-331-3p_478323_mir & -5.93 & $3.27 \mathrm{E}-03$ & $7.90 \mathrm{E}-03$ & 0.715 & 0.656 & 0.774 & 0.761 & 0.775 & 0.744 \\
\hline hsa-let-7f-2-3p_477843_mir & -3.26 & 1.19E-02 & $2.33 \mathrm{E}-02$ & 0.715 & 0.655 & 0.776 & 0.682 & 0.567 & 0.814 \\
\hline hsa-miR-195-3p_478744_mir & -4.54 & $6.67 \mathrm{E}-03$ & $1.40 \mathrm{E}-02$ & 0.712 & 0.652 & 0.773 & 0.717 & 0.712 & 0.723 \\
\hline hsa-miR-378a-5p_478076_mir & -5.30 & $2.12 \mathrm{E}-03$ & $5.41 \mathrm{E}-03$ & 0.71 & 0.65 & 0.769 & 0.723 & 0.584 & 0.882 \\
\hline hsa-miR-1-3p_477820_mir & -6.27 & $6.10 \mathrm{E}-03$ & $1.31 \mathrm{E}-02$ & 0.706 & 0.644 & 0.768 & 0.732 & 0.778 & 0.679 \\
\hline hsa-miR-615-3p_478175_mir & -3.14 & $4.99 E-03$ & $1.13 E-02$ & 0.706 & 0.643 & 0.769 & 0.696 & 0.652 & 0.746 \\
\hline hsa-miR-545-3p_479002_mir & -2.99 & $1.99 \mathrm{E}-02$ & $3.66 \mathrm{E}-02$ & 0.704 & 0.64 & 0.767 & 0.675 & 0.678 & 0.672 \\
\hline hsa-miR-548a-3p_478157_mir & -3.75 & 4.83E-03 & $1.10 \mathrm{E}-02$ & 0.703 & 0.641 & 0.765 & 0.716 & 0.572 & 0.881 \\
\hline hsa-miR-1248_478653_mir & -2.64 & $1.08 \mathrm{E}-02$ & $2.17 \mathrm{E}-02$ & 0.702 & 0.642 & 0.763 & 0.707 & 0.764 & 0.643 \\
\hline hsa-miR-381-3p_477816_mir & -5.10 & 5.97E-03 & $1.31 \mathrm{E}-02$ & 0.701 & 0.639 & 0.763 & 0.734 & 0.727 & 0.742 \\
\hline hsa-miR-627-5p_478427_mir & -4.94 & $5.01 \mathrm{E}-04$ & $1.62 \mathrm{E}-03$ & 0.701 & 0.632 & 0.77 & 0.735 & 0.863 & 0.589 \\
\hline hsa-miR-1301-3p_477897_mir & -5.30 & $5.52 \mathrm{E}-03$ & $1.22 \mathrm{E}-02$ & 0.696 & 0.638 & 0.754 & 0.711 & 0.596 & 0.843 \\
\hline hsa-miR-486-3p_478422_mir & -6.30 & $1.86 \mathrm{E}-04$ & $7.18 \mathrm{E}-04$ & 0.692 & 0.626 & 0.758 & 0.725 & 0.891 & 0.535 \\
\hline hsa-miR-200a-3p_478490_mir & -4.37 & $1.79 E-02$ & $3.39 E-02$ & 0.691 & 0.626 & 0.756 & 0.719 & 0.792 & 0.635 \\
\hline hsa-miR-15a-3p_477928_mir & -3.53 & $6.65 E-03$ & $1.40 E-02$ & 0.688 & 0.62 & 0.755 & 0.703 & 0.808 & 0.584 \\
\hline hsa-miR-548 g-3p_479020_mir & -2.12 & $2.25 \mathrm{E}-02$ & 4.04E-02 & 0.687 & 0.625 & 0.75 & 0.691 & 0.759 & 0.613 \\
\hline hsa-miR-432-5p_478101_mir & -3.66 & $2.70 E-02$ & 4.65E-02 & 0.683 & 0.621 & 0.746 & 0.691 & 0.724 & 0.652 \\
\hline hsa-miR-15b-5p_478313_mir & -6.53 & $2.89 \mathrm{E}-02$ & $4.90 \mathrm{E}-02$ & 0.682 & 0.619 & 0.745 & 0.666 & 0.692 & 0.637 \\
\hline hsa-miR-132-5p_478705_mir & -6.01 & $3.23 E-03$ & 7.86E-03 & 0.68 & 0.614 & 0.745 & 0.734 & 0.867 & 0.582 \\
\hline hsa-let-7e-5p_478579_mir & -5.40 & $2.04 \mathrm{E}-02$ & $3.72 \mathrm{E}-02$ & 0.679 & 0.614 & 0.744 & 0.655 & 0.556 & 0.769 \\
\hline hsa-miR-485-5p_478126_mir & -2.09 & $2.46 \mathrm{E}-02$ & $4.30 E-02$ & 0.678 & 0.613 & 0.743 & 0.688 & 0.734 & 0.634 \\
\hline
\end{tabular}


Table 2 (continued)

\begin{tabular}{llllllllll}
\hline miRNA & LogFC & p-value & Adj.p-value & AUC & $\begin{array}{l}\text { AUC 95\% } \\
\text { Cl_lower }\end{array}$ & $\begin{array}{l}\text { AUC 95\% } \\
\text { Cl_upper }\end{array}$ & Accuracy & Sensitivity & Specificity \\
\hline hsa-miR-299-5p_478793_mir & -5.05 & $8.70 \mathrm{E}-04$ & $2.62 \mathrm{E}-03$ & 0.672 & 0.603 & 0.741 & 0.702 & 0.858 & 0.524 \\
hsa-miR-16-1-3p_478727_mir & -4.44 & $1.55 \mathrm{E}-03$ & $4.13 \mathrm{E}-03$ & 0.669 & 0.603 & 0.735 & 0.699 & 0.841 & 0.537 \\
hsa-miR-215-5p_478516_mir & -7.23 & $8.19 \mathrm{E}-05$ & $3.88 \mathrm{E}-04$ & 0.668 & 0.601 & 0.735 & 0.718 & 0.896 & 0.514 \\
hsa-miR-103a-2-5p_477864_mir & -4.32 & $2.05 \mathrm{E}-02$ & $3.73 \mathrm{E}-02$ & 0.664 & 0.603 & 0.724 & 0.664 & 0.529 & 0.818 \\
hsa-miR-29a-5p_478002_mir & -6.48 & $2.54 \mathrm{E}-03$ & $6.26 \mathrm{E}-03$ & 0.643 & 0.568 & 0.717 & 0.723 & 0.896 & 0.524 \\
hsa-miR-874-3p_478205_mir & -2.80 & $2.77 \mathrm{E}-02$ & $4.74 \mathrm{E}-02$ & 0.638 & 0.575 & 0.701 & 0.617 & 0.524 & 0.722 \\
hsa-miR-502-5p_478954_mir & -4.03 & $2.18 \mathrm{E}-02$ & $3.93 \mathrm{E}-02$ & 0.637 & 0.573 & 0.701 & 0.662 & 0.53 & 0.813 \\
hsa-miR-542-5p_478337_mir & -5.28 & $3.71 \mathrm{E}-03$ & $8.83 \mathrm{E}-03$ & 0.633 & 0.564 & 0.702 & 0.68 & 0.855 & 0.481 \\
hsa-miR-362-3p_478058_mir & -5.81 & $1.73 \mathrm{E}-03$ & $4.56 \mathrm{E}-03$ & 0.632 & 0.564 & 0.701 & 0.69 & 0.879 & 0.475 \\
hsa-miR-431-3p_478888_mir & -4.55 & $4.63 \mathrm{E}-03$ & $1.06 \mathrm{E}-02$ & 0.624 & 0.552 & 0.696 & 0.678 & 0.853 & 0.478 \\
hsa-miR-192-3p_478741_mir & -4.08 & $5.15 \mathrm{E}-03$ & $1.16 \mathrm{E}-02$ & 0.61 & 0.535 & 0.685 & 0.674 & 0.85 & 0.474 \\
hsa-miR-589-5p_479073_mir & -2.81 & $2.28 \mathrm{E}-02$ & $4.07 \mathrm{E}-02$ & 0.582 & 0.512 & 0.652 & 0.633 & 0.795 & 0.448 \\
hsa-miR-888-5p_479192_mir & -3.79 & $2.06 \mathrm{E}-02$ & $3.73 \mathrm{E}-02$ & 0.559 & 0.489 & 0.63 & 0.656 & 0.861 & 0.422 \\
hsa-miR-15b-3p_477929_mir & -3.73 & $1.76 \mathrm{E}-02$ & $3.37 \mathrm{E}-02$ & 0.529 & 0.459 & 0.599 & 0.629 & 0.86 & 0.365 \\
hsa-miR-651-5p_479131_mir & -3.94 & $2.90 \mathrm{E}-02$ & $4.90 \mathrm{E}-02$ & 0.523 & 0.448 & 0.599 & 0.638 & 0.87 & 0.372 \\
\hline
\end{tabular}

Log fold-change expression, p-value, adjusted p-value, AUC values, accuracy, sensitivity, specificity, and $95 \%$ of confidence intervals of the 210 dysregulated miRNAs $\mathrm{Cl}$ confidence of interval

profile of serum samples from CRC patients and healthy controls and identified 16 miRNA that were expressed in a significantly higher levels among CRC patients. Of these, 7 miRNAs (let-7a, miR-1229, miR1246, miR-150, miR-21, miR-223, and miR-23a) were suggested as promising diagnostic biomarkers of CRC with an AUC between 0.67 and 0.95. More recently, the serum exosomal miRNA-19a was found to be upregulated in the serum of CRC patients compared to healthy volunteers, but also was associated with poor prognosis [19]. Finally, Zhao et al. [20], demonstrated that the exosomal miRNA-21 expression is associated with the early diagnosis of CRC. Although plasma and serum have reported promising biomarkers for CRC diagnosis, other approaches as it is the use of proximal bodily fluids as a source of biomarkers have aroused the attention of the biomarker research community. Proximal bodily fluids, such as urine for prostate cancer [21], or uterine fluid for endometrial cancer [22] have demonstrated that this type of fluids offers an improved representation of the molecular alterations that takes place in the tumor. The peritoneal lavage is a proximal fluid with an unexploded value in biomarker research for cancers originating within the peritoneal cavity. Tokuhisa et al. [23] showed that EV-associated miRNAs can be consistently extracted from this bodily fluid and that miRNAs expression profiles can indicate the status of peritoneum in gastric cancer patients.

To the best of our knowledge, our study is the first to report the value of this proximal fluid for the identification of miRNAs associated to EVs in CRC. Importantly, this study unveiled the promising use of the top-10 miRNA dysregulated (miRNA-199b-5p, miRNA-150-5p, miRNA-29c-5p, miRNA-218-5p, miRNA-99a-3p, miRNA-383-5p, miRNA-199a-3p, miRNA-193a-5p, miRNA-10b-5p and miRNA-181c-5p) as diagnostic biomarkers, all showing the AUC value higher than 0.95. Those biomarkers should be validated as well as combined in order to increase the already excellent accuracy of individual miRNAs. However, this should be done 


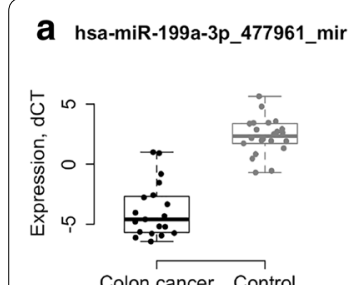

Colon cancer Control

hsa-miR-10b-5p_478494_mir
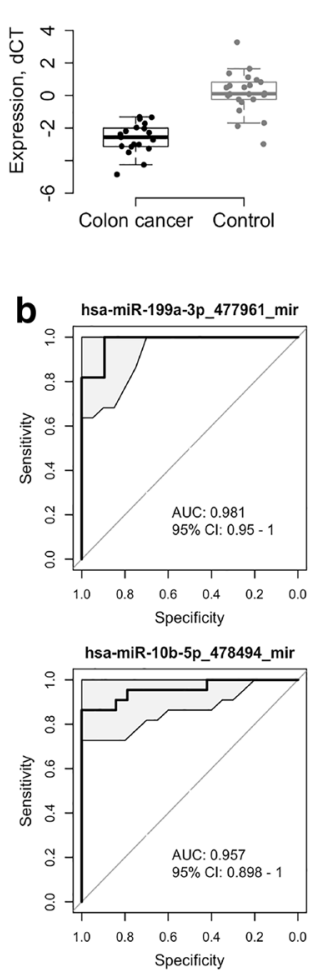

hsa-miR-150-5p_477918_mir

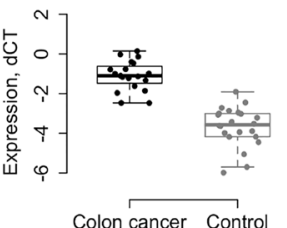

Colon cancer Control

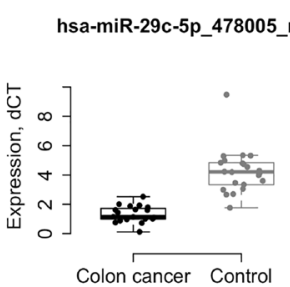

Colon cancer Control

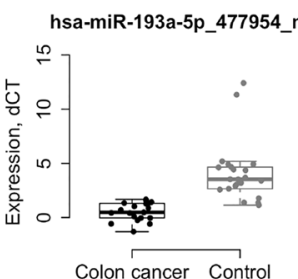

Colon cancer Control
hsa-miR-99a-3p_479224_mir

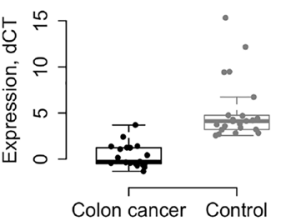

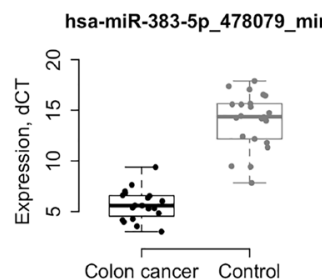
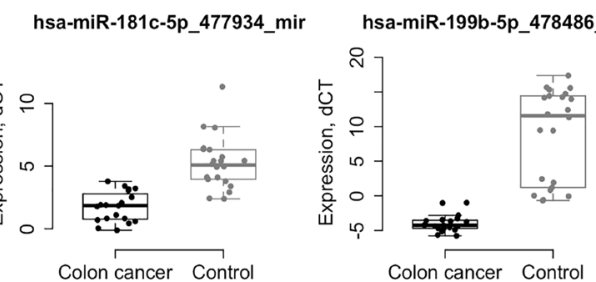

hsa-miR-218-5p_477977_mir
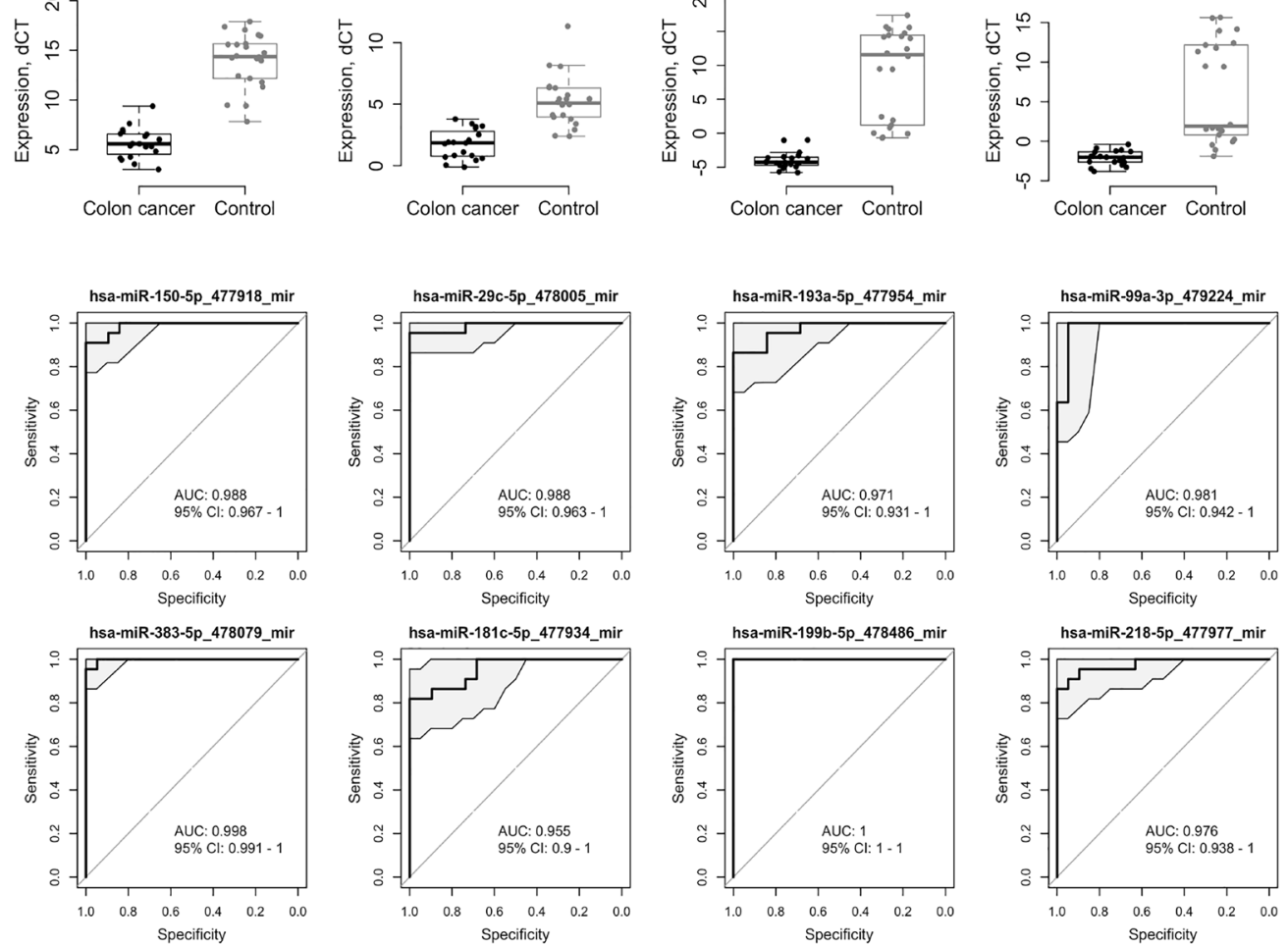

Fig. 1 Diagnostic performance of the top-10 differentially expressed miRNAs. a Relative dCT values of top differentially expressed miRNAs in patients with CRC $(n=19)$ compared to control patients $(n=22) .{ }^{* *} p<0.05$. b ROC-curves and AUC-scores the top-10 differentially expressed miRNAs

Table 3 Published studies of the top-10 miRNAs dysregulated in CRC patients

\begin{tabular}{lll}
\hline & Tissue samples & Other type of samples \\
\hline miR-199b-5p & Not previously reported & Not previously reported \\
miR-150-5p & Upregulated: [24, 25] & Downregulated: serum [28] \\
miR-29c-5p & Downregulated: $[26,27]$ & Not previously reported \\
miR-218-5p & Not previously reported & Not previously reported \\
& Upregulated: [29] & Not previously reported \\
miR-99a-3p & Downregulated: [30, 31] & Not previously reported \\
miR-383-5p & Not previously reported & Upregulated in stool [33, 34] \\
miR-199a-3p & Downregulated: [32] & Not previously reported \\
miR-193a-5p & Upregulated: [26] & Not previously reported \\
miR-10b-5p & Downregulated in CRC cell lines [35] & Not previously reported \\
\hline miR-181c-5p & Downregulated: [30, 36, 37] & Upregulated: [38]
\end{tabular}




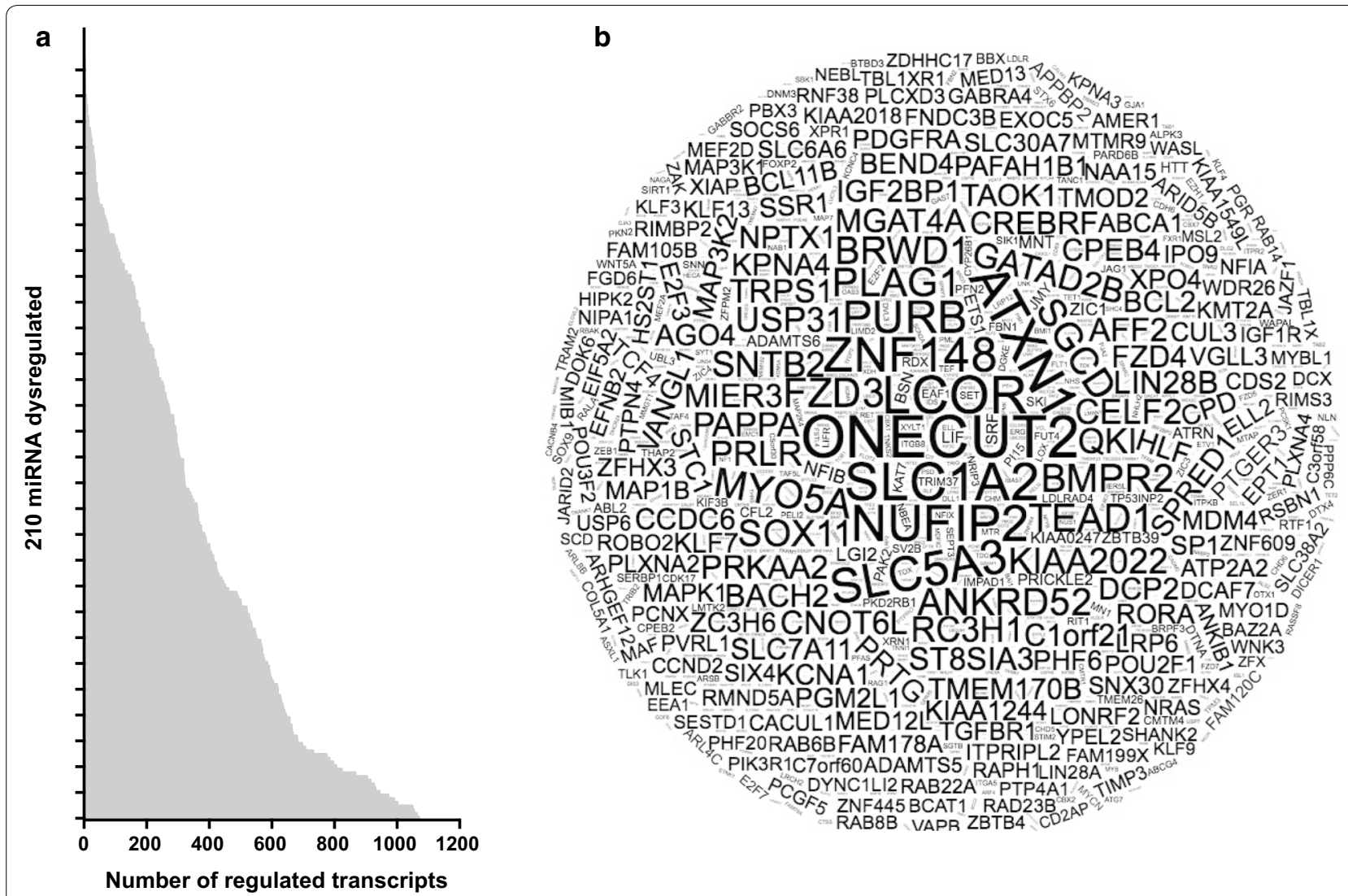

Fig. 2 Prediction of miRNA target transcripts. a Boxplot of the number of transcripts regulated by the 210 dysregulated miRNAs. b Graphical representation in which the transcript ID is represented with a different font size accordingly to the times that is predicted to be regulated by the different miRNAs, i.e. the most frequently regulated transcripts are shown in a larger font size

in an independent study including a larger cohort of patients. Moreover, further analysis should be performed to elucidate the prognostic value of the detection of the different types of miRNAs in EVs isolated from peritoneal lavages.

\section{Conclusions}

In this study, we have demonstrated that use of EVassociated miRNA of ascitic liquid from control patients and peritoneal lavages from CRC patients are an untapped source of biomarkers. Specifically, we identified miRNA-199b-5p, miRNA-150-5p, miRNA-29c-5p, miRNA-218-5p, miRNA-99a-3p, miRNA-383-5p, miRNA-199a-3p, miRNA-193a-5p, miRNA-10b-5p and miRNA-181c-5p as promising biomarkers of CRC diagnosis with the AUC value higher than 0.95 . 


\section{a GO-Biological Process}

- Metabolic process (24.3\% - 2408 genes) Biosynthetic process $(29.6 \%)$ Organic substance metabolic process $(29.5 \%)$ Cellular metabolic process $(22.7 \%)$ Primary metabolic process $(7.5 \%)$

- Biological regulation (22.5\% - 2239 genes) Regulation of biological process $(80 \%)$ Regulation of biological quality $(11.2 \%)$ Regulation of molecular function (8.8\%)

- Cellular process (10.7\% - 1064 genes) Signal transduction (26\%) Cellular component organization (24\%) Cellular metabolic process (16.5\%) Cellular developmental process $(7.1 \%)$ Cell cycle $(4.6 \%)$

- Cellular component organization or biogenesis (10.4\% - 1039 genes) Cellular component organization (98.7\%) Cellular component biogénesis $(1.3 \%)$

- Localization (9.9\% - 983 genes) Macromolecule localization (13.8\%) Establishment of localization (76.2\%)

- Developmental process (6\% - 601 genes) Cellular developmental process $(43.8 \%)$ Anatomical structure development $(32.9 \%)$

- Response to stimulus (4.6\% - 455 genes) Response to chemical $(33.6 \%)$ Response to stress (25.4\%)

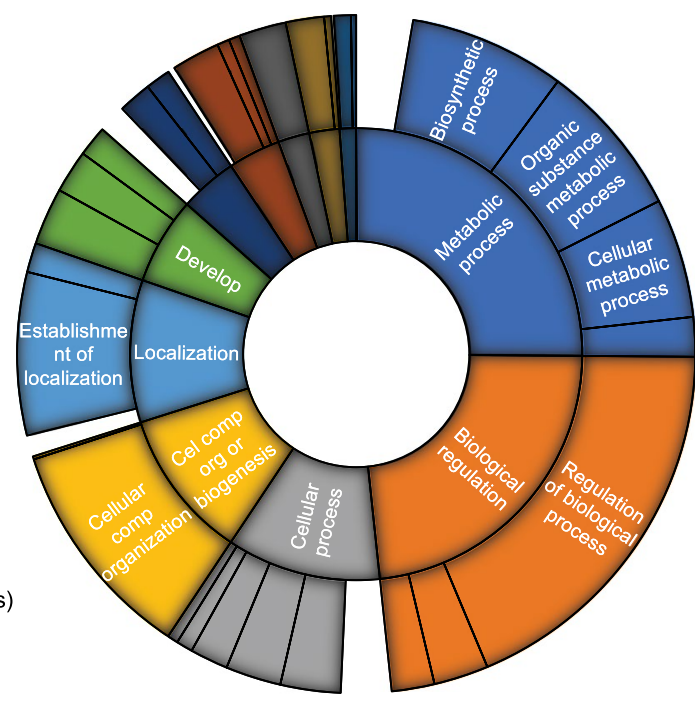

- Multicellular organismal process (3.6\% -362 genes) System process $(61.5 \%)$ Multicellular organism development $(15.6 \%)$ Pattern specification process (14.2\%)

- Biological adhesión (2.2\% - 217 genes) Cell adhesión (100\%)

- Signaling $(2.1 \%-208$ genes $)$ Cell-cell signaling $(15 \%)$ Signal transduction (85\%)

\author{
(1.1\% - 114 genes) \\ Immune response $(69.5 \%)$ \\ Leukocyte activation $(21 \%)$
}

- Immune system process

- Others (2.7\% - 266 genes)

\section{b GO-Molecular Function}

- Binding (37.8\% - 2682 genes) lon binding $(7.4 \%)$

Organic cyclic compound binding (27.4\%) Protein binding (43.3\%)

V Catalytic activity (31.2\% - 2217 genes) Catalytic activity, acting on a protein $(31.5 \%)$ Hydrolase activity (28.7\%) Ttransferase activity (17.5\%)

- Transcription regulator activity (9.4\% - 668 genes) Transcription coregulator activity (17.6\%) DNA-binding transcription factor activity $(82.4 \%)$

- Transporter activity $(7.5 \%-531$ genes) Transmembrane transporter activity (82\%) Lipid transporter activity (10.3\%)

- Molecular transducer activity ( $5.7 \%$ - 408 genes) Signaling receptor activity $(89 \%)$

- Molecular function regulator (5.3\% - 378 genes) Enzyme regulator activity (83.2\%) Channel regulator activity (5.35\%) Guanyl-nucleotide exchange factor activity (10.5\%)

- Structural molecule activity $(2.5 \%-179$ genes $)$ Structural constituent of ribosome $(40 \%)$ Extracellular matrix structural constituent (14.1\%) Protein-containing complex scaffold activity $(30.8 \%)$ Structural constituent of nuclear pore $(15.4 \%)$

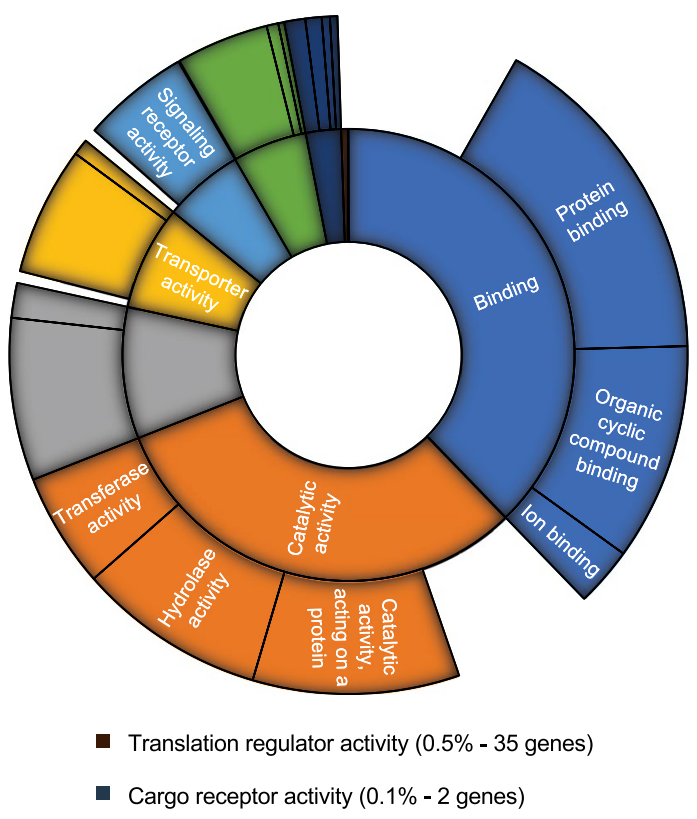

Fig. 3 Sun projection plot of GO terms. Predicted transcripts regulated by the differentially expressed miRNAs. a GO analysis of up-regulated and down-regulated target genes according to biological process. b GO analysis of up-regulated and down-regulated target genes according to molecular function 


\section{Additional files}

Additional file 1: Table S1. Clinicopathological characteristics of all patients.

Additional file 2: Figure S1. Workflow. Workflow of the study design.

Additional file 3: Figure S2. EVs characterization. (A) Box-plot representing the average mode of EVs isolated from the peritoneal lavage and ascitic fluid of CRC and control patients, respectively (Mean \pm SD); measured by Nanoparticle Tracking Analysis. (B) Size distribution and concentration of isolated EVs of a peritoneal lavage of a CRC patient (left) and a ascitic fluid of a control patient (right), measured by Nanoparticle Tracking Analysis.

\section{Abbreviations}

CRC: colorectal cancer; EVs: extracellular vesicles; RT: reverse transcription; Ct: cycle threshold; FDR: False Discovery Rate; AUC: area under the ROC curve; GO: Gene Ontology; BP: biological process; MF: molecular function; 3'-UTR: 3'-untranslated region.

\section{Acknowledgements}

The authors would like to acknowledge the work of all who have participated in the recruitment of clinical samples. We also thank the patients for their willingness to participate in the study.

\section{Authors' contributions}

Study conception and design: XMG, EC. Sample and data collection: SG, MRM, JT, JMP, AGM, JES, MR. Acquisition of data: BRC, CM, SB, EG. Analysis and interpretation of data: SB, JP, CM, BRC, JMFP, EG, XMG, EC. Drafting of manuscript: BRC, CM. Critical revision: CM, JMFP, EG, JMP, JP, XMG, EC. All authors read and approved the final manuscript.

\section{Funding}

EC hold a postdoctoral fellowship from the Departament de Salut of the Generalitat de Catalunya (SLT002/16/00274). This work was supported by grants: Discovery, validation and implementation of biomarkers for Precision Oncology (ISCIII PIE15/00029), CIBERONC (CB16/12/00231 and CB16/12/00328). Grups consolidats de la Generalitat de Catalunya (2017SGR1368 and 2017SGR1661). Work supported by IRBLleida BIOBANK (B.0000682) and Plataforma biobancos PT17/0015/0027.

\section{Availability of data and materials}

All bioinformatics analysis was performed with the BioConductor (version 3.7) [10] project in the R statistical environment (version 3.5.0).

\section{Ethics approval and consent to participate}

Participants in the study attended to the Hospital Arnau de Vilanova in Lleida, Spain. The Clinical Research Ethics Committee of the hospital approved the study and all the participating patients provided a signed informed consent.

\section{Consent for publication}

All authors consent for publication.

\section{Competing interests}

The authors declare that they have no competing interests.

\section{Author details}

${ }^{1}$ Department of Pathology and Molecular Genetics/Oncologic Pathology Group, Biomedical Research Institute of Lleida (IRBLleida), University of Lleida, CIBERONC, Lleida, Spain. ${ }^{2}$ Department of Pathology, University Hospital of Bellvitge, Bellvitge Biomedical Research Institute (IDIBELL), L'Hospitalet de Llobregat, Barcelona, Spain. ${ }^{3}$ Universitat Autònoma de Barcelona (UAB), Barcelona, Spain. ${ }^{4}$ Biomedical Research Group in Gynecology, Vall Hebron Research Institute (VHIR), Universitat Autònoma de Barcelona, CIBERONC, Pg. Vall Hebron 119-129, 08035 Barcelona, Spain. ${ }^{5}$ Centre for Genomic Regulation (CRG), The Barcelona Institute or Science and Technology, Dr. Aiguader 88, Barcelona 08003, Spain. ${ }^{6}$ Department of Surgery, Hospital Arnau de Vilanova, Biomedical Research Institute of Lleida (IRBLleida), Lleida, Spain. ${ }^{7}$ Exosomes Laboratory and Metabolomics Platform, CIC bioGUNE, CIBEREHD Bizkaia
Technology Park, Derio, Spain. ${ }^{8}$ Pleural Medicine Unit, Arnau de Vilanova University Hospital, IRBLleida, Lleida, Spain. ${ }^{9}$ Gynecological Oncology Department, Vall Hebron University Hospital, CIBERONC, Barcelona, Spain. ${ }^{10}$ IKERBASQUE, Basque Foundation for Science, 48011 Bilbao, Spain. ${ }^{11}$ University Pompeu Fabra, Barcelona, Spain. ${ }^{12}$ Oncologic Pathology Group, Department of Medicine UdL, Biomedical Research Institute of Lleida (IrbLleida), Av. Rovira Roure 80, 25198 Lleida, Spain.

Received: 2 April 2019 Accepted: 13 June 2019

Published online: 20 June 2019

\section{References}

1. Bray F, Ferlay J, Soerjomataram I, Siegel RL, Torre LA, Jemal A. Global cancer statistics 2018: GLOBOCAN estimates of incidence and mortality worldwide for 36 cancers in 185 countries. CA Cancer J Clin. 2018:68(6):394-424.

2. Siegel RL, Miller KD, Jemal A. Cancer statistics, 2018: cancer statistics, 2018. CA Cancer J Clin. 2018;68(1):7-30.

3. Schmoll HJ, Van Cutsem E, Stein A, Valentini V, Glimelius B, Haustermans $\mathrm{K}$, et al. ESMO Consensus Guidelines for management of patients with colon and rectal cancer. A personalized approach to clinical decision making. Ann Oncol. 2012;23(10):2479-516.

4. Ogata-Kawata H, Izumiya M, Kurioka D, Honma Y, Yamada Y, Furuta K, et al. Circulating exosomal microRNAs as biomarkers of colon cancer. PLoS ONE. 2014;9(4):e92921.

5. Wang $Y$, Chen Z, Chen W. Novel circulating microRNAs expression profile in colon cancer: a pilot study. Eur J Med Res. 2017. https://doi. org/10.1186/s40001-017-0294-5.

6. Zeng $M$, Zhu L, Li L, Kang C. miR-378 suppresses the proliferation, migration and invasion of colon cancer cells by inhibiting SDAD1. Cell Mol Biol Lett. 2017. https://doi.org/10.1186/s11658-017-0041-5.

7. Yuan Q, Cao G, Li J, Zhang Y, Yang W. MicroRNA-136 inhibits colon cancer cell proliferation and invasion through targeting liver receptor homolog-1/Wnt signaling. Gene. 2017;628:48-55.

8. Yáñez-Mó M, Siljander P-RM, Andreu Z, Zavec A, Borràs FE, Buzas El, et al. Biological properties of extracellular vesicles and their physiological functions. J Extracell Vesicles. 2015;4(1):27066.

9. Campoy I, Lanau L, Altadill T, Sequeiros T, Cabrera S, Cubo-Abert M, et al. Exosome-like vesicles in uterine aspirates: a comparison of ultracentrifugation-based isolation protocols. J Transl Med. 2016;14:1. https://doi. org/10.1186/s12967-016-0935-4.

10. Huber W, Carey VJ, Gentleman R, Anders S, Carlson M, Carvalho BS, et al. Orchestrating high-throughput genomic analysis with bioconductor. Nat Methods. 2015;12(2):115-21.

11. R Foundation for Statistical Computing, Vienna, Austria. R: A language and environment for statistical computing.

12. Dvinge $H$, Bertone P. HTqPCR: high-throughput analysis and visualization of quantitative real-time PCR data in R. Bioinformatics. 2009;25(24):3325-6.

13. Ritchie ME, Phipson B, Wu D, Hu Y, Law CW, Shi W, et al. limma powers differential expression analyses for RNA-sequencing and microarray studies. Nucleic Acids Res. 2015;43(7):e47.

14. BENJAMINIt Y, Hochberg Y. Controlling the false discovery rate: a practical and powerful approach to multiple testing. 13.

15. Dweep H, Gretz N. miRWalk2.0: a comprehensive atlas of microRNAtarget interactions. Nat Methods. 2015;12(8):697.

16. Mi H, Muruganujan A, Casagrande JT, Thomas PD. Large-scale gene function analysis with the PANTHER classification system. Nat Protoc. 2013:8(8):1551-66.

17. Mazeh H, Mizrahi I, llyayev N, Halle D, Brücher BL, Bilchik A, et al. The diagnostic and prognostic role of microrna in colorectal cancer-a comprehensive review. J Cancer. 2013;4(3):281-95.

18. Chen $X, B a Y, M a L$, Cai X, Yin Y, Wang K, et al. Characterization of microRNAs in serum: a novel class of biomarkers for diagnosis of cancer and other diseases. Cell Res. 2008;18(10):997-1006.

19. Matsumura T, Sugimachi K, linuma H, Takahashi Y, Kurashige J, Sawada G, et al. Exosomal microRNA in serum is a novel biomarker of recurrence in human colorectal cancer. Br J Cancer. 2015;113(2):275-81. 
20. Zhao L, Yu J, Wang J, Li H, Che J, Cao B. Isolation and Identification of miRNAs in exosomes derived from serum of colon cancer patients. J Cancer. 2017:8(7):1145-52.

21. Sequeiros T, Rigau M, Chiva C, Montes M, Garcia-Grau I, Garcia M, et al. Targeted proteomics in urinary extracellular vesicles identifies biomarkers for diagnosis and prognosis of prostate cancer. Oncotarget. 2017; 8 (3). http://www.oncotarget.com/fulltext/13634.

22. Martinez-Garcia E, Lesur A, Devis L, Cabrera S, Matias-Guiu X, Hirschfeld $M$, et al. Targeted proteomics identifies proteomic signatures in liquid biopsies of the endometrium to diagnose endometrial cancer and assist in the prediction of the optimal surgical treatment. Clin Cancer Res. 2017;23(21):6458-67.

23. Tokuhisa M, Ichikawa Y, Kosaka N, Ochiya T, Yashiro M, Hirakawa K, et al. Exosomal miRNAs from peritoneum lavage fluid as potential prognostic biomarkers of peritoneal metastasis in gastric cancer. PLoS ONE. 2015;10(7):e0130472

24. Volinia S, Calin GA, Liu C-G, Ambs S, Cimmino A, Petrocca F, et al. A microRNA expression signature of human solid tumors defines cancer gene targets. Proc Natl Acad Sci. 2006;103(7):2257-61.

25. Jacob H, Stanisavljevic L, Storli KE, Hestetun KE, Dahl O, Myklebust MP. Identification of a sixteen-microRNA signature as prognostic biomarker for stage II and III colon cancer. Oncotarget. 2017;8 (50). http://www. oncotarget.com/fulltext/21237. Accessed 25 Feb 2019.

26. Slattery ML, Wolff E, Hoffman MD, Pellatt DF, Milash B, Wolff RK. MicroRNAs and colon and rectal cancer: differential expression by tumor location and subtype. Genes Chromosomes Cancer. 2011;50(3):196-206.

27. Ma Y, Zhang P, Wang F, Zhang H, Yang J, Peng J, et al. miR-150 as a potential biomarker associated with prognosis and therapeutic outcome in colorectal cancer. Gut. 2012;61(10):1447-53.

28. Wang $Q$, Huang Z, Ni S, Xiao X, Xu Q, Wang L, et al. Plasma miR-601 and miR-760 are novel biomarkers for the early detection of colorectal cancer. PLoS ONE. 2012;7(9):e44398.

29. Zhou X, Huang $X$, Liang S, Tang S, Wu S, Huang T, et al. Identifying miRNA and gene modules of colon cancer associated with pathological stage by weighted gene co-expression network analysis. OncoTargets Ther. 2018;11:2815-30.

30. Mosakhani N, Sarhadi VK, Borze I, Karjalainen-Lindsberg M-L, Sundström J, Ristamäki R, et al. MicroRNA profiling differentiates colorectal cancer according to KRAS status. Genes Chromosomes Cancer. 2012;51(1):1-9.
31. Ye Y, Gu B, Wang Y, Shen S, Huang W, et al. E2F1-mediated MNX1-AS1miR-218-5p-SEC61A1 feedback loop contributes to the progression of colon adenocarcinoma. J Cell Biochem. 2019;120(4):6145-53.

32. Hamfjord J, Stangeland AM, Hughes T, Skrede ML, Tveit KM, Ikdahl T, et al. Differential expression of miRNAs in colorectal cancer: comparison of paired tumor tissue and adjacent normal mucosa using high-throughput sequencing. PLoS ONE. 2012;7(4):e34150.

33. Ahmed FE, Ahmed NC, Gouda MM, Vos PW, Bonnerup C. RT-qPCR for fecal mature microRNA quantification and validation. In: Beaulieu J-F, editor. Colorectal cancer. New York: Springer; 2018. p. 203-15. https://doi. org/10.1007/978-1-4939-7765-9_13.

34. Ahmed FE, Ahmed NC, Vos PW, Bonnerup C, Atkins JN, Casey M, et al. Diagnostic MicroRNA markers to screen for sporadic human colon cancer in stool: I. Proof of principle. Cancer Genomics. 2013;21:93.

35. Shirafkan N, Shomali N, Kazemi T, Shanehbandi D, Ghasabi M, Baghbani E, et al. MicroRNA-193a-5p inhibits migration of human HT-29 colon cancer cells via suppression of metastasis pathway. J Cell Biochem. 2018. https:// doi.org/10.1002/jcb.28164.

36. Arndt GM, Dossey L, Cullen LM, Lai A, Druker R, Eisbacher M, et al. Characterization of global microRNA expression reveals oncogenic potential of miR-145 in metastatic colorectal cancer. BMC Cancer. 2009. https://doi. org/10.1186/1471-2407-9-374.

37. Sarver AL, French AJ, Borralho PM, Thayanithy V, Oberg AL, Silverstein $\mathrm{KA}$, et al. Human colon cancer profiles show differential microRNA expression depending on mismatch repair status and are characteristic of undifferentiated proliferative states. BMC Cancer. 2009. https://doi. org/10.1186/1471-2407-9-401.

38. Monzo M, Navarro A, Bandres E, Artells R, Moreno I, Gel B, et al. Overlapping expression of microRNAs in human embryonic colon and colorectal cancer. Cell Res. 2008;18(8):823-33.

\section{Publisher's Note}

Springer Nature remains neutral with regard to jurisdictional claims in published maps and institutional affiliations.
Ready to submit your research? Choose BMC and benefit from:

- fast, convenient online submission

- thorough peer review by experienced researchers in your field

- rapid publication on acceptance

- support for research data, including large and complex data types

- gold Open Access which fosters wider collaboration and increased citations

- maximum visibility for your research: over $100 \mathrm{M}$ website views per year

At BMC, research is always in progress.

Learn more biomedcentral.com/submissions 